\title{
The phase of the Daubechies filters
}

\section{Djalil Kateb and Pierre Gilles Lemarié-Rieusset}

Abstract. We give the first term of the asymptotic development for the phase of the $N$-th (minimum-phased) Daubechies filter as $N$ goes to $+\infty$. We obtain this result through the description of the complex zeros of the associated polynomial of degree $2 N+1$.

\section{Introduction.}

The Daubechies filters $m_{N}(\xi)$ are defined in the following way [2]:

i) $m_{N}(\xi)$ is a trigonometric polynomial of degree $2 N+1$

$$
m_{N}(\xi)=\sum_{k=0}^{2 N+1} a_{N, k} e^{-i k \xi}
$$

with real-valued coefficients $a_{N, k}$.

ii) $\sqrt{2} m_{N}(\xi)$ and $\sqrt{2} e^{-i \xi} \bar{m}_{N}(\xi+\pi)$ are conjugate quadrature filters

$$
\left|m_{N}(\xi)\right|^{2}+\left|m_{N}(\xi+\pi)\right|^{2}=1 .
$$

iii) $m_{N}(\xi)$ satisfies at 0 and $\pi$

$$
\begin{gathered}
m_{N}(0)=1 \\
\frac{\partial^{p}}{\partial \xi^{p}} m_{N}(\pi)=0, \quad \text { for } p \in\{0,1, \ldots, N\} .
\end{gathered}
$$


The importance of those filters is due to the following facts: the associated wavelet $\psi_{N}$ defined by

$$
\hat{\psi}_{N}(\xi)=e^{-i \xi / 2} \bar{m}_{N}\left(\frac{\xi}{2}+\pi\right) \prod_{j=2}^{+\infty} m_{N}\left(\frac{\xi}{2^{j}}\right)
$$

generates an orthonormal basis of $L^{2}(\mathbb{R})\left\{2^{j / 2} \psi_{N}\left(2^{j} x-k\right)\right\}_{j \in \mathbb{Z}, k \in \mathbb{Z}}$ and satisfies the cancellation properties

$$
\int x^{p} \psi_{N}(x) d x=0, \quad \text { for } p \in\{0,1, \cdots, N\}
$$

and has a support of minimal length among all orthonormal wavelets satisfying (6).

Conditions (1) to (4) don't define $m_{N}$ in an unique way. As a matter of fact, there is exactly $2^{[(N+1) / 2]}$ solutions $m_{N}$ (where $[x]$ is the integer part of $x$ ). Indeed, conditions (1) to (4) determine only the modulus of $m_{N}$

$$
\begin{gathered}
\left|m_{N}(\xi)\right|^{2}=Q_{N}(\cos \xi) \\
Q_{N}(X)=\left(\frac{1+X}{2}\right)^{N+1} \sum_{k=0}^{N}\left(\begin{array}{c}
N+k \\
k
\end{array}\right)\left(\begin{array}{c}
1-X \\
2
\end{array}\right)^{k} .
\end{gathered}
$$

We are going to check easily the following result on the roots of $Q_{N}$.

Proposition 1. The roots of $Q_{N}$ are $X=-1$ with multiplicity $N+1$ and $N$ roots $X_{N, 1}, \cdots, X_{N, N}$ with multiplicity 1 such that

i) for $1 \leq k \leq N$, $\operatorname{Re} X_{N, k}>0$ and $X_{N, N+1-k}=\overline{X_{N, k}}$,

ii) for $1 \leq k \leq[N / 2]$, $\operatorname{Im} X_{N, k}>0$,

iii) if $N$ is odd, $X_{N,(N+1) / 2}>1$.

With help of Proposition 1, we may easily describe the solutions $m_{N}$ of (1) to (4). Indeed, if $X_{N, k}=\left(z_{N, k}+1 / z_{N, k}\right) / 2$ with $\left|z_{N, k}\right|>1$, then we have

$$
m_{N}(\xi)=\prod_{k=1}^{[(N+1) / 2]} S_{N, k}(\xi)\left(\frac{1+e^{-i \xi}}{2}\right)^{N+1}
$$


where, for $1 \leq k \leq[N / 2]$,

$$
\begin{aligned}
& S_{N, k}(\xi)=\frac{\left(e^{-i \xi}-z_{N, k}\right)\left(e^{-i \xi}-\bar{z}_{N, k}\right)}{\left|1-z_{N, k}\right|^{2}} \\
& \text { or } \quad S_{N, k}(\xi)=\frac{\left(1-z_{N, k} e^{-i \xi}\right)\left(1-\bar{z}_{N, k} e^{-i \xi}\right)}{\left|1-z_{N, k}\right|^{2}} .
\end{aligned}
$$

If $N$ is odd,

$$
\begin{aligned}
& S_{N,(N+1) / 2}(\xi)=\frac{e^{-i \xi}-z_{N,(N+1) / 2}}{1-z_{N,(N+1) / 2}} \\
& \text { or } \quad S_{N,(N+1) / 2}(\xi)=\frac{1-z_{N,(N+1) / 2} e^{-i \xi}}{1-z_{N,(N+1) / 2}} .
\end{aligned}
$$

The case where all the roots of $M_{N}(z)$ (the polynomial such that $\left.m_{N}(\xi)=M_{N}\left(e^{-i \xi}\right)\right)$ are outside the unit disk is the minimum-phased Daubechies filter

$$
m_{N}(\xi)=\left(\frac{1+e^{-i \xi}}{2}\right)^{N+1} \prod_{k=1}^{N} \frac{e^{-i \xi}-z_{N, k}}{1-z_{N, k}}
$$

The aim of this paper is to describe the phase of the Daubechies filters as $N$ goes to $+\infty$. Indeed, the modulus of $m_{N}$ is described by (7) and (8) and one easily checks that

$$
\lim _{N \rightarrow+\infty}\left|m_{N}(\xi)\right|= \begin{cases}1, & \text { if }|\xi|<\frac{\pi}{2}, \\ \frac{1}{\sqrt{2}}, & \text { if }|\xi|=\frac{\pi}{2} \\ 0, & \text { if } \frac{\pi}{2}<|\xi| \leq \pi .\end{cases}
$$

The phase of $m_{N}$, on the other hand, is much more delicate to study: it depends of course on the choice of the factors $S_{N, k}$ in (9), but even for the case of minimum-phased filters we are not aware of any previous results on the behaviour of the phase.

We are going to give an approximate value of $z_{N, k}$ which allows the determination of the phase of $m_{N}$. More precisely, if $Z_{1}, \ldots, Z_{N}$ are $N$ complex numbers such that for $k \in\{1, \ldots, N\},\left|Z_{k}\right| \neq 1$ and if

$$
\Pi\left(Z_{1}, \ldots, Z_{N}\right)(\xi)=\prod_{k=1}^{N} \frac{e^{-i \xi}-Z_{k}}{1-Z_{k}},
$$


we define the phase $\omega\left(Z_{1}, \ldots, Z_{N}\right)(\xi)$ as the $C^{\infty}$ real-valued function such that $\omega(0)=0$ and

$$
\Pi\left(Z_{1}, \ldots, Z_{N}\right)(\xi)=\prod_{k=1}^{N}\left|\frac{e^{-i \xi}-Z_{k}}{1-Z_{k}}\right| e^{-i \omega\left(Z_{1}, \ldots, Z_{N}\right)(\xi)}
$$

This function is easily computed as

$$
\omega\left(Z_{1}, \ldots, Z_{N}\right)(\xi)=\operatorname{Im}\left(\int_{0}^{\xi} \sum_{k=1}^{N} \frac{i e^{-i s}}{e^{-i s}-Z_{k}} d s\right) .
$$

Theorem 1. Let $Q_{N}(X)$ be given by $(8), X_{N, 1}, \ldots, X_{N, N}$ be its roots which are not equal to -1 ordered by:

- for $1 \leq k \leq[(N+1) / 2], \operatorname{Im} X_{N, k} \geq 0$ and $X_{N, N+1-k}=\overline{X_{N, k}}$,

- $\left|X_{N, 1}\right|<\left|X_{N, 2}\right|<\cdots<\left|X_{N,[(N+1) / 2]}\right|$

and let $z_{N, k}$ be defined by $X_{N, k}=\left(z_{N, k}+1 / z_{N, k}\right) / 2$ and $\left|z_{N, k}\right|>1$.

For $1 \leq k \leq N$, we approximate $z_{N, k}$ by $Z_{N, k}$ where:

i) for $1 \leq k \leq\left[\left(N^{1 / 5}\right) / \log N\right], Z_{N, k}=i-\overline{\gamma_{k}} / \sqrt{N}$, where $\gamma_{1}, \gamma_{2}$, $\ldots, \gamma_{k}, \ldots$ are the roots of $\operatorname{erfc}(z)=1-(2 / \sqrt{\pi}) \int_{0}^{z} e^{-s^{2}} d s$, such that $\operatorname{Im} \gamma_{k}>0$ and ordered by $\left|\gamma_{1}\right|<\left|\gamma_{2}\right|<\cdots<\left|\gamma_{k}\right|<\ldots$, where

ii) for $\left[\left(N^{1 / 5}\right) / \log N\right]<k \leq[(N+1) / 2], Z_{N, k}=\theta_{N, k}+\sqrt{\theta_{N, k}^{2}-1}$,

$$
\operatorname{Im} \theta_{N, k}>0
$$

$$
1-\theta_{N, k}^{2}=\left(1+\frac{1}{N} \log \left(2 \sqrt{2 N \pi \sin \varphi_{N, k}}\right)\right) e^{-2 i \varphi_{N, k}}
$$

and

$$
\varphi_{N, k}=\frac{8 k-1}{8 N+6} \pi
$$

iii) for $[(N+1) / 2]<k \leq N, Z_{N, k}=\bar{Z}_{N, N+1-k}$.

Then for any choice

$$
m_{N}(\xi)=\left(\frac{1+e^{-i \xi}}{2}\right)^{N+1} \Pi\left(z_{N, 1}^{\varepsilon_{1}}, \ldots, z_{N, N}^{\varepsilon_{N}}\right)(\xi)
$$


of the Daubechies filter $m_{N}$ (where $\varepsilon_{k}= \pm 1$ and $\varepsilon_{N+1-k}=\varepsilon_{k}$ ), the approximation

$$
\tilde{m}_{N}(\xi)=\left(\frac{1+e^{-i \xi}}{2}\right)^{N+1} \Pi\left(Z_{N, 1}^{\varepsilon_{1}}, \ldots, Z_{N, N}^{\varepsilon_{N}}\right)(\xi)
$$

satisfies

$$
\left|\omega\left(z_{N, 1}^{\varepsilon_{1}}, \ldots, z_{N, N}^{\varepsilon_{N}}\right)(\xi)-\omega\left(Z_{N, 1}^{\varepsilon_{1}}, \ldots, Z_{N, N}^{\varepsilon_{N}}\right)(\xi)\right| \leq C_{0} \frac{(\log N)^{2}}{N^{1 / 5}}
$$

for all $\xi \in \mathbb{R}$, where $C_{0}$ doesn't depend neither on $N \geq 2$ nor on $\xi$ nor on the $\varepsilon_{k}$ 's.

Thus, due to Theorem 1, we may give the phase of $m_{N}$ with an $o$ (1) precision! Of course, we need the knowledge of the roots of the complementary error function; these roots are described in [3] and our results give again the same estimates, as we shall see.

We may greatly simplify the approximating $Z_{N, k}$ 's if we accept to get a greater error. For instance, we may characterize easily the minimum-phased filters with an $O(\sqrt{N})$ error:

Theorem 2. Let

$$
m_{N}(\xi)=\left(\frac{1+e^{-i \xi}}{2}\right)^{N+1} \Pi\left(z_{N, 1}, \ldots, z_{N, N}\right)(\xi)
$$

be the N-th minimum-phased Daubechies filter. Then the phase

$$
\omega\left(z_{N, 1}, \ldots, z_{N, N}\right)(\xi)
$$

satisfies

$$
\left|\omega\left(z_{N, 1}, \ldots, z_{N, N}\right)(\xi)-N \omega(\xi)\right| \leq C_{0} \sqrt{N}, \quad \text { for all } \xi \in \mathbb{R}
$$

where $C_{0}$ doesn't depend on $\xi$ nor on $N$ and where

$$
\omega(\xi)=\frac{1}{2 \pi}\left(\operatorname{Li}_{2}(-\sin \xi)-\operatorname{Li}_{2}(\sin \xi)\right)=\frac{-1}{\pi} \sum_{k=0}^{+\infty} \frac{(\sin \xi)^{2 k+1}}{(2 k+1)^{2}}
$$


The $L i_{2}$ function is the polylogarithm of order 2

$$
\operatorname{Li}_{2}(z)=\sum_{k=1}^{\infty} \frac{z^{k}}{k^{2}}=\int_{0}^{z} \frac{1}{u} \log \frac{1}{1-u} d u .
$$

The function $\left(\mathrm{Li}_{2}(z)-\mathrm{Li}_{2}(-z)\right) / 2$ is known under the name of Legendre's $\chi_{2}$ function.

Theorem 2 will be proved by approximating $m_{N}$ by

$$
\tilde{m}_{N}(\xi)=\left(\frac{1+e^{-i \xi}}{2}\right)^{N+1} \pi\left(\tilde{Z}_{N, 1}, \ldots, \tilde{Z}_{N, N}\right)(\xi)
$$

with

$$
\tilde{Z}_{N, k}=\sqrt{e^{-i \theta_{N, k}}}+\sqrt{1+e^{-i \theta_{N, k}}}, \quad \theta_{N, k}=-\pi+\frac{16 k-2}{8 N+6} \pi,
$$

Then $\omega\left(\tilde{Z}_{N, 1}, \ldots, \tilde{Z}_{N, N}\right) / N$ is identified with a Riemann sum for the integral

$$
\frac{1}{2 \pi} \operatorname{Im} \int_{-\pi}^{\pi} \log \frac{1}{\sqrt{e^{-i \theta}}+\sqrt{1+e^{-i \theta}}-e^{-i \xi}} d \theta=\omega(\xi) .
$$

This approximating $\tilde{Z}_{N, k}$ is a simplified version of the approximating $Z_{N, k}$ of Theorem 1, obtained by neglecting the term

$$
\frac{1}{N} \log 2 \sqrt{2 N \pi \sin \varphi_{N, k}} .
$$

We will be also able to give a description of a family of almost linearphased Daubechies filters:

Theorem 3. Let

$$
m_{N}(\xi)=\left(\frac{1+e^{-i \xi}}{2}\right)^{N+1} \pi\left(z_{N, 1}^{\varepsilon_{N, 1}}, \ldots, z_{N, N}^{\varepsilon_{N, N}}\right)(\xi)
$$

be the $N$-th Daubechies filter with $N=4 q$ and with the following choice of $\varepsilon_{N, k}$ : for $1 \leq p \leq q, \varepsilon_{N, 4 p-3}=\varepsilon_{N, 4 p}=1$ and $\varepsilon_{N, 4 p-2}=\varepsilon_{4 p-1}=$ -1 (so that $\left.\varepsilon_{N, N+1-k}=\varepsilon_{N, k}\right)$. Then the phase $\omega\left(z_{N, 1}^{\varepsilon_{N, 1}}, \ldots, z_{N, N}^{\varepsilon_{N, N}}\right)(\xi)$ satisfies:

$$
\left|\omega\left(z_{N, 1}^{\varepsilon_{N, 1}}, \ldots, z_{N, N}^{\varepsilon_{N, N}}\right)(\xi)-\frac{1}{2} N \xi\right| \leq C_{0}, \quad \text { for all } \xi \in \mathbb{R}
$$


where $C_{0}$ doesn't depend on $\xi$ nor on $N$.

We are now going to prove Theorem 1 (and obtain theorems 2 and 3 as corollaries). Of course, it amounts to give a precise description of the $\operatorname{roots} X_{N, k}$ of $Q_{N}(X)$. If we neglect the term $\log 2 \sqrt{2 N \pi \sin \varphi_{N, k}} / N$ in $Z_{N, k}$, we obtain as a first approximation that the $z_{N, k}$ are close to the arc $\{|z-1|=\sqrt{2}$, Re $z \geq 0\}$ (which can be parameterized as $\left\{\sqrt{e^{-i \theta}}+\sqrt{1+e^{-i \theta}},-\pi \leq \theta \leq \pi\right\}$ ), or equivalently that the $X_{N, k}$ are close to the half-lemniscate $\left\{\left|1-X_{N, k}^{2}\right|=1, \operatorname{Re} X_{N, k} \geq 0\right\}$. This will be obtained by representing $Q_{N}(X)$ as a Bernstein polynomial on $[-1,1]$ approximating the piecewise analytical function $\chi_{[0,1]}$

$$
Q_{N}(X)=\sum_{k=N+1}^{2 N+1}\left(\begin{array}{c}
2 N+1 \\
k
\end{array}\right)\left(\frac{1+X}{2}\right)^{k}\left(\frac{1-X}{2}\right)^{2 N+1-k}
$$

(a formula pointed by many authors [1], [6], [11]). In that form, $Q_{N}(X)$ corresponds to a Herrmann filter [4] and it is precisely the figure in Herrmann's paper representing the $z_{N, k}$ 's for $Q_{21}$ which lead us to conjecture the behaviour of the $z_{N, k}$ 's.

A classical theorem of Kantorovitch [5], [7] on the behaviour of Bernstein polynomials of piecewise analytical functions ensures that $Q_{N}(X)$ converges to 0 uniformly on any compact subset of the interior of the half lemniscat $\left\{\left|1-x^{2}\right|<1\right.$, Re $\left.x<0\right\}$ and to 1 uniformly on any compact subset of $\left\{\left|1-x^{2}\right|<1\right.$, Re $\left.x>0\right\}$. We will use similar tools to study $Q_{N}(X)$ outside of the convergence subsets.

Near the critical point $X=0$, the approximation by points on the lemniscat is no longer precise enough, and we will show that for the small roots $X_{N, k},-\sqrt{N} X_{N, k}$ is to be approximated by a root of the complementary error function. Such an approximation occurs for instance in the study of the (spurious) zeros of the Taylor polynomials of the exponential function [12] and we will use quite similar tools to get our description. The main difference, however, is maybe that we are dealing with a divergent family of polynomials.

Notations. We will define as usually $\log z$ and $\sqrt{z}$ as the reciprocal functions of

$$
\begin{gathered}
\left.z=\log w \in\{z \in \mathbb{C}:|\operatorname{Im} z|<\pi\} \longmapsto w=e^{z} \in\{w \in \mathbb{C}: w \notin(-\infty, 0])\right\} \\
z=\sqrt{w} \in\{z \in \mathbb{C}: \operatorname{Re} z>0\} \longmapsto w=z^{2} \in\{w \in \mathbb{C}: w \notin(-\infty, 0]\}
\end{gathered}
$$


The paper will be organized in the following way:

1. $Q_{N}$ as a Bernstein polynomial and other preliminary results.

2. Small roots of $Q_{N}$ : first estimates.

3. Big roots of $Q_{N}$ : first estimates.

4. Big roots of $Q_{N}$ : further estimates.

5. Small roots of $Q_{N}$ : further estimates.

6. The phase of a general Daubechies filter.

7. Minimum-phased Daubechies filters.

8. Almost linear-phased Daubechies filters.

1. $Q_{N}$ as a Bernstein polynomial and other preliminary results.

We begin by proving a first localization result:

Result 1. For $N \geq 2$ and $t \neq-1$, if $Q_{N}(t)=0$ then $|1-t|<1$.

Proof. This will be the only time where we use the Daubechies formula (8) for $Q_{N}(X)$. This formula gives that if $Q_{N}(t)=0$ and $t \neq-1$, then

$$
\sum_{k=0}^{N} \frac{1}{2^{k}}\left(\begin{array}{c}
N+k \\
k
\end{array}\right)(1-t)^{k}=0 \text {. }
$$

If we define $\alpha_{k}$ as $\alpha_{k}=\left(\begin{array}{c}N+k \\ k\end{array}\right) / 2^{k}, 0 \leq k \leq N$, then we have obviously $0<\alpha_{0}<\alpha_{1}<\cdots<\alpha_{N-1}=\alpha_{N}$, and we may apply a very classical lemma of Eneström, Kakeya and Hurwirtz (quoted by G. Pólya and Szegö [10, Exercise III-22]):

Lemma 1. If $0<a_{0}<a_{1}<\cdots<a_{N-1}=a_{N}$ and if $\sum_{k=0}^{N} a_{k} s^{k}=0$ then $|s|<1$.

Proof OF The LeMma. If $s \geq 0$ then $\sum_{k=0}^{N} a_{k} s^{k}>0$; if $s \notin[0,+\infty)$, then

$$
\left|a_{0}+\sum_{k=1}^{N}\left(a_{k}-a_{k-1}\right) s^{k}\right|<a_{0}+\sum_{k=1}^{N}\left(a_{k}-a_{k-1}\right)|s|^{k},
$$

thus if $|s| \geq 1$ (so that $|s|^{k} \leq|s|^{N+1}$ ) and $s \notin[0,+\infty$ ), we get

$$
\left|(1-s) \sum_{k=0}^{N} a_{k} s^{k}\right|>|s|^{N+1}\left(a_{N}-\sum_{k=1}^{N}\left(a_{k}-a_{k-1}\right)-a_{0}\right)=0 .
$$


Thus, we have shown that the roots $t$ of $Q_{N}$ such that $t \neq-1$ are located in the open disk of radius 1 and of center 1 , and that the associated values $1-t^{2}$ are located in the interior of a cardioid.

From now until the end, we will use formula (22) instead of formula (8) to represent $Q_{N}$. The main interest in the representation of $Q_{N}$ as a Bernstein polynomial is that $Q_{N}$ is easily differentiated: (22) gives

$$
\frac{d}{d t} Q_{N}(t)=\frac{(2 N+1) !}{4^{N}(N !)^{2}} \frac{1}{2}\left(1-t^{2}\right)^{N} .
$$

This expression can be easily related to the expression of $Q_{N}(\cos \xi)$ given by Y. Meyer ([8])

$$
\begin{aligned}
Q_{N}(\cos \xi) & =\int_{-1}^{\cos \xi} \frac{(2 N+1) !}{4^{N}(N !)^{2}} \frac{1}{2}\left(1-t^{2}\right)^{N} d t \\
& =\int_{\xi}^{\pi} \frac{(2 N+1) !}{4^{N}(N !)^{2}} \frac{1}{2}(\sin \theta)^{2 N+1} d \theta
\end{aligned}
$$

We will use intensively formula (24) in the following. If $t$ is small, we approximate $Q_{N}(t)$ by $Q_{N}(0)=1 / 2$ and obtain

$$
Q_{N}(t)=\frac{1}{2}\left(1+\frac{(2 N+1) !}{4^{N}(N !)^{2}} \int_{0}^{t}\left(1-s^{2}\right)^{N} d s\right)
$$

while for a bigger $t$ (with $\operatorname{Re} t>0$ ) we approximate $Q_{N}(t)$ by $Q_{N}(1)=1$ and obtain

$$
Q_{N}(t)=1-\frac{1}{2} \frac{(2 N+1) !}{4^{N}(N !)^{2}} \int_{t}^{1}\left(1-s^{2}\right)^{N} d s
$$

Stirling's formula $N !=(N / e)^{N} \sqrt{2 \pi N}\left(1+1 /(12 N)+O\left(1 / N^{2}\right)\right)$ allows one to simplify formulas $(25)$ and (26)

$$
\frac{(2 N+1) !}{4^{N}(N !)^{2}}=2 \sqrt{\frac{N}{\pi}}\left(1+O\left(\frac{1}{N^{2}}\right)\right) .
$$

Thus $Q_{N}(t)=0$ may be rewritten as

(28) $1+\frac{2}{\sqrt{\pi}} \int_{0}^{\sqrt{N} t}\left(1-\frac{s^{2}}{N}\right)^{N} d s=1-2 \frac{\sqrt{N}}{\sqrt{\pi}} \frac{4^{N}(N !)^{2}}{(2 N+1) !}=O\left(\frac{1}{N^{2}}\right)$ 
or as

$$
\sqrt{N} \int_{t}^{1}\left(1-s^{2}\right)^{N} d s=2 \frac{4^{N}(N !)^{2}}{(2 N+1) !}=\sqrt{\pi}+O\left(\frac{1}{N^{2}}\right) .
$$

Formula (28) will be used for the small roots (sections 2 and 5) and formula (29) for the big roots (sections 3 and 4).

We mention a further application of (24) (which will not be used in the following): we may compute explicitly the generating series for $Q_{N}(t)$ when $\operatorname{Re} t<0$ :

Proposition 2. Assume that $\operatorname{Re} t<0$ and $\left|\left(1-t^{2}\right) u\right|<1$. Then

$$
\sum_{N=0}^{+\infty} Q_{N}(t) u^{N}=\frac{1}{2} \frac{1-t^{2}}{\sqrt{1-u\left(1-t^{2}\right)}\left(-t+\sqrt{1-u\left(1-t^{2}\right)}\right)} .
$$

Proof. We differentiate $\sum_{N=0}^{+\infty} Q_{N}(t) u^{N}$ with respect to $t$. Then (24) gives

$$
\begin{aligned}
\frac{\partial}{\partial t}\left(\sum_{N=0}^{+\infty} Q_{N}(t) u^{N}\right) & =\sum_{N=0}^{+\infty} \frac{1}{2} \frac{(2 N+1) !}{4^{N} N !} \frac{\left(\left(1-t^{2}\right) u\right)^{N}}{N !} \\
& =\frac{1}{2}\left(1-u\left(1-t^{2}\right)\right)^{-3 / 2}
\end{aligned}
$$

hence

$$
\sum_{N=0}^{+\infty} Q_{N}(t) u^{N}=\int_{-1}^{t} \frac{1}{2} \frac{d s}{\left(1-\left(1-s^{2}\right) u\right)^{3 / 2}} .
$$

On the other hand, if we differentiate $t /\left(1-u\left(1-t^{2}\right)\right)^{1 / 2}$, we get

$$
\frac{\partial}{\partial t}\left(\frac{t}{\left(1-u\left(1-t^{2}\right)\right)^{1 / 2}}\right)=\frac{1-u\left(1-t^{2}\right)-t^{2} u}{\left(1-u\left(1-t^{2}\right)\right)^{3 / 2}}=\frac{1-u}{\left(1-u\left(1-t^{2}\right)\right)^{3 / 2}} .
$$

Thus we have

$$
\begin{aligned}
\sum_{N=0}^{+\infty} Q_{N}(t) u^{N} & =\frac{1}{2(1-u)}\left(\frac{t}{\left(1-u\left(1-t^{2}\right)\right)^{1 / 2}}+1\right) \\
& =\frac{1}{2(1-u)} \frac{1-u\left(1-t^{2}\right)-t^{2}}{\left(1-u\left(1-t^{2}\right)\right)^{1 / 2}\left(\left(1-u\left(1-t^{2}\right)\right)^{1 / 2}-t\right)} \\
& =\frac{1}{2} \frac{1-t^{2}}{\left(1-u\left(1-t^{2}\right)\right)^{1 / 2}\left(\left(1-u\left(1-t^{2}\right)\right)^{1 / 2}-t\right)}
\end{aligned}
$$


As a corollary, we get:

Result 2. If $t \in \mathbb{C}$ is such that $\left|1-t^{2}\right|>1$, then

$$
\limsup _{N \rightarrow+\infty}\left|Q_{N}(t)\right|=+\infty
$$

ProOF. If Ret $t<0$, this is obvious by formula (30); the right-hand term of equality (30) has $1 /\left|1-t^{2}\right|$ as its radius of convergence in $u$, so that

$$
\limsup _{N \rightarrow+\infty}\left|Q_{N}(t)\right|^{1 / N}=\left|1-t^{2}\right| .
$$

If $\operatorname{Re} t>0$, then $Q_{N}(t)=1-Q_{N}(-t)$ so that again

$$
\limsup _{N \rightarrow+\infty}\left|Q_{N}(t)\right|^{1 / N}=\left|1-t^{2}\right|
$$

If $\operatorname{Re} t=0$ and $t \neq 0$, then

$$
\left|Q_{N}(t)\right| \sim \frac{1}{2} 2 \sqrt{\frac{N}{\pi}} \int_{0}^{|t|}\left(1+\rho^{2}\right)^{N} d \rho \longrightarrow+\infty, \quad \text { as } N \longrightarrow+\infty .
$$

A last (and direct) application of formula (24) is Proposition 1.

\section{Result 3.}

i) If $t$ is a root of $Q_{N}(t)$ and $t \neq-1$, then $t$ has multiplicity 1.

ii) If $N$ is even, $t=-1$ is the unique real root of $Q_{N}$.

iii) If $N$ is odd, $Q_{N}$ has only one other real root $x_{N,(N+1) / 2} \neq-1$, and $x_{N,(N+1) / 2}>1$.

Proof. By (24), we know that the only roots of $d Q_{N} / d t$ are 1 and -1 , so i) is obvious. Moreover, if $N$ is even, $d Q_{N} / d t$ is non-negative on $\mathbb{R}$ and thus $Q_{N}$ is increasing: -1 is the unique real root of $Q_{N}$. If $N$ is odd, then $Q_{N}$ decreases on $(-\infty,-1]$, vanishes at -1 , increases between -1 and 1 , and decreases again from the value 1 at $t=1$ to the value $-\infty$ at $t=+\infty: Q_{N}$ has another real root $x_{N,(N+1) / 2}>1$.

Results 1 and 3 imply obviously Proposition 1. 


\section{Small roots of $Q_{N}$ : first estimates.}

In this section, we are going to prove the following result:

Result 4. Let $\varepsilon_{0} \in(0,1 / 2)$ and $K=\left[\varepsilon_{0} \log N /(2 \pi)\right]$. Then, if $N$ is big enough, the number of roots $t$ of $Q_{N}(t)$ such that $\operatorname{Im} t \geq 0$ and $|t| \leq \sqrt{2 K \pi / N}$ is exactly $K$. Moreover, if we list those roots as $x_{N, 1}, \ldots, x_{N, K}$ with $\left|x_{N, k}\right|<\left|x_{N, k+1}\right|$ and fix $\varepsilon_{1} \in\left(\varepsilon_{0}, 1 / 2\right)$, we have

$$
\left|x_{N, k}+\frac{1}{\sqrt{N}} \bar{\gamma}_{k}\right| \leq C\left(\varepsilon_{0}, \varepsilon_{1}\right) \frac{1}{\sqrt{N} N^{1-2 \varepsilon_{1}}},
$$

where $\gamma_{1}, \ldots, \gamma_{K}$ are the $K$ first roots $\gamma$ of $\operatorname{erfc}(\gamma)=0$ with $\operatorname{Im} \gamma \geq 0$.

Proof. Assume that $|t| \leq \sqrt{\alpha_{1} \log N / N}$ for some fixed $\alpha_{1}>0$. Then, using formulas (25) and (27), we write

$$
Q_{N}(t)=\left(\frac{1}{2}+\eta_{N}\right)\left(1+\eta_{N}^{\prime}+\frac{2}{\sqrt{\pi}} \int_{0}^{\sqrt{N} t}\left(1-\frac{s^{2}}{N}\right)^{N} d s\right)
$$

where $\eta_{N}, \eta_{N}^{\prime}$ are two constants (depending only on $N$ ) which are $O\left(1 / N^{2}\right)$. Now, if $|u| \leq \sqrt{\alpha_{1} \log N}$, we have

$$
\frac{\left|u^{4}\right|}{N} \leq \alpha_{1}^{2} \frac{(\log N)^{2}}{N}=o(1)
$$

hence one may find $C_{0} \geq 0$ so that for $N$ big enough $\left(N \geq N_{0}\right.$ where $N_{0}$ depends only on $\alpha_{1}$ )

$$
\left|\left(1-\frac{u^{2}}{N}\right)^{N}-e^{-u^{2}}\right| \leq C_{0}\left|e^{-u^{2}} \frac{u^{4}}{N}\right| \leq C_{0} \alpha_{1}^{2} \frac{(\log N)^{2}}{N^{1-\alpha_{1}}} .
$$

Hence we get for fixed $\alpha_{1}>0$ and for $N \geq N_{0}\left(\alpha_{1}\right)$

$$
\left|\left(\frac{1}{2}+\eta_{N}\right)^{-1} Q_{N}(t)-\operatorname{erfc}(-\sqrt{N} t)\right| \leq C_{1} \frac{(\log N)^{5 / 2}}{N^{1-\alpha_{1}}},
$$

for $|t| \leq \sqrt{\alpha_{1} \log N / N}$, where $C_{1}$ depends only on $\alpha_{1}$.

Now, assume that $\theta$ is such that $Q_{N}(\theta)=0$ or $\operatorname{erfc}(-\sqrt{N} \theta)=0$ and that $|\theta| \leq \sqrt{\alpha_{1} \log N / N}$; in every case we have

$$
|\operatorname{erfc}(-\sqrt{N} \theta)| \leq C_{1} \frac{(\log N)^{5 / 2}}{N^{1-\alpha_{1}}} .
$$


We are going to show that for $\delta_{0}$ small enough, $\operatorname{erfc}(-\sqrt{N} \theta+z)$ is not too small on $|z|=\delta_{0}$. Indeed we have

$$
\begin{aligned}
|\operatorname{erfc}(-\sqrt{N} \theta+z)-\operatorname{erfc}(-\sqrt{N} \theta)| & =\frac{2}{\sqrt{\pi}}\left|\int_{0}^{z} e^{-N \theta^{2}} e^{2 \sqrt{N} \theta s} e^{-s^{2}} d s\right| \\
& \geq \frac{1}{2} \frac{2}{\sqrt{\pi}}\left|e^{-N \theta^{2}}\right||z| \geq \frac{1}{\sqrt{\pi}} N^{-\alpha_{1}}|z|
\end{aligned}
$$

provided that

$$
|z| \leq \min \left\{2 \sqrt{\alpha_{1} \log N}, \frac{1}{8 C_{2} \sqrt{\alpha_{1} \log N}}\right\},
$$

where $C_{2}=\max _{|w| \leq 1}\left|\left(e^{w}-1\right) / w\right|$.

Thus, if $|\theta| \leq \sqrt{\alpha_{2} \log N / N}$, where $\alpha_{2}<\alpha_{1}<1 / 2$, and if $N$ is big enough so that

$$
\sqrt{\alpha_{2} \frac{\log N}{N}}+\frac{1}{8 C_{2} \sqrt{\alpha_{1} N \log N}}<\sqrt{\alpha_{1} \frac{\log N}{N}}
$$

and

$$
C_{1} \sqrt{\pi} \frac{(\log N)^{5 / 2}}{N^{1-2 \alpha_{1}}}<\frac{1}{8 C_{2} \sqrt{\alpha_{1} \log N}}<2 \sqrt{\alpha_{1} \log N},
$$

we obtain that $Q_{N}(t)$ and $\operatorname{erfc}(-\sqrt{N} t)$ have the same number of zeros inside the open disk $D\left(\theta, C_{1} \sqrt{\pi}(\log N)^{5 / 2} / N^{3 / 2-2 \alpha_{1}}\right)$ (by Rouché's theorem).

In order to conclude, we need some information on the zeros of $\operatorname{erfc}(z)$. A theorem by Fettis, Cuslin and Cramer ([3]) gives a development of $\gamma_{k}$

$$
\begin{aligned}
& \gamma_{k}=e^{3 i \pi / 4}\left(\sqrt{\left(2 k-\frac{1}{4}\right) \pi}\right. \\
&-\frac{i}{2 \sqrt{\left(2 k-\frac{1}{4}\right) \pi}} \log \left(2 \sqrt{\pi} \sqrt{\left(2 k-\frac{1}{4}\right) \pi}\right) \\
&+\left.O\left(\frac{(\log k)^{2}}{k \sqrt{k}}\right)\right) .
\end{aligned}
$$

Thus if $M_{0}$ is a fixed number in $(-\pi / 4,3 \pi / 4)$, the number of roots $\gamma$ of $\operatorname{erfc}(\gamma)=0$ such that $\operatorname{Im} \gamma \geq 0$ and $|\gamma| \leq \sqrt{2 k \pi+M_{0}}$ is exactly $k$ when $k$ is large enough. 
Now we may prove Result 4 . Let $\varepsilon_{0}<1 / 2$ and $K=\left[\varepsilon_{0} \log N /(2 \pi)\right]$. For each root $t$ of $Q_{N}(s)$ such that $|\operatorname{Im} t| \geq 0$ and $|t| \leq \sqrt{2 K \pi / N} \leq$ $\sqrt{\varepsilon_{0} \log N / N}$ there is a root $\theta$ of $\operatorname{erfc}(-\sqrt{N} s)$ such that

$$
|\theta-t| \leq C_{1} \sqrt{\pi} \frac{(\log N)^{5 / 2}}{N^{3 / 2-2 \varepsilon_{1}}}
$$

(where $\varepsilon_{0}<\varepsilon_{1}<1 / 2$ and $N \geq N_{1}\left(\varepsilon_{1}\right)$ ). Then we have

$$
\begin{aligned}
|\sqrt{N} \theta| & \leq \sqrt{2 K \pi}+C_{1} \sqrt{\pi} \frac{(\log N)^{5 / 2}}{N^{1-2 \varepsilon_{1}}} \\
& \leq \sqrt{2 K \pi}+\frac{\pi}{16 \sqrt{2 K \pi}} \\
& \leq \sqrt{\left(2 K+\frac{1}{8}\right) \pi}
\end{aligned}
$$

provided that $N \geq N_{2}\left(\varepsilon_{1}\right)$. But we know that there are exactly $2 K$ roots of $\operatorname{erfc}(-\sqrt{N} s)$ inside the disk $D(0, \sqrt{(2 K+1 / 4) \pi} / \sqrt{N})$. Conversely, if $\theta$ is a root of $\operatorname{erfc}(-\sqrt{N} s)$ such that

$$
|\theta| \leq \sqrt{\frac{2 K \pi}{N}}-C_{1} \sqrt{\pi} \frac{(\log N)^{5 / 2}}{N^{3 / 2}-2 \varepsilon_{1}} \leq \sqrt{\varepsilon_{0} \frac{\log N}{N}},
$$

there is a root $t$ of $Q_{N}(s)$ such that

$$
|\theta-t| \leq C_{1} \sqrt{\pi} \frac{(\log N)^{5 / 2}}{N^{3 / 2-2 \varepsilon_{1}}}
$$

hence $|t| \leq \sqrt{2 K \pi / N}$; moreover for $N \geq N_{2}\left(\varepsilon_{1}\right)$ we have

$$
\sqrt{2 K \pi}-C_{1} \sqrt{\pi} \frac{(\log N)^{5 / 2}}{N^{1-2 \varepsilon_{1}}}>\sqrt{2 K \pi}-\frac{\pi}{16 \sqrt{2 K \pi}}>\sqrt{\left(2 K-\frac{1}{8}\right) \pi},
$$

so that we have again $2 K$ roots of $\operatorname{erfc}(-\sqrt{N} s)$ such that

$$
|\theta| \leq \sqrt{\frac{2 K \pi}{N}}-C_{1} \sqrt{\pi} \frac{(\log N)^{5 / 2}}{N^{1-2 \varepsilon_{1}}} .
$$

Finally, we conclude by noticing that (33) shows us that if $\operatorname{erfc}\left(-\sqrt{N} \theta_{i}\right)$ $=0, i=1,2, \theta_{1} \neq \theta_{2}$ and $\left|\theta_{i}\right| \leq \sqrt{(2 K+1 / 8) \pi / N}$ then $\left|\theta_{1}-\theta_{2}\right| \geq$ 
$C_{0} / \sqrt{K N}$ and $\left|\operatorname{Im} \theta_{i}\right| \geq C_{0} \sqrt{K / N}$ for some positive $C_{0}$ which doesn't depend on $K$ nor $N$; hence the balls

$$
D\left(\theta_{i}, C_{1} \sqrt{\pi} \frac{(\log N)^{5 / 2}}{N^{3 / 2-2 \varepsilon_{1}}}\right)
$$

are disjoint and don't meet the real axis (for $N$ large enough). Thus (31) is proved, if we notice that

$$
\frac{(\log N)^{5 / 2}}{N^{1-2 \varepsilon_{1}}}<\frac{1}{N^{1-2 \varepsilon_{1}^{\prime}}}
$$

for $\varepsilon_{1}<\varepsilon_{1}^{\prime}<1 / 2$ and $N$ large enough.

\section{Big roots of $Q_{N}$ : first estimates.}

In this section, we are going to devote our attention to formula (26). A straigthforward application of (26) is the following one:

Result 5. For $N$ large enough, if $t \neq-1$ and $Q_{N}(t)=0$, then $\left|1-t^{2}\right|>1$.

Proof. If $Q_{N}(t)=0$, then we have $\sqrt{N} \int_{t}^{1}\left(1-s^{2}\right)^{N} d s=\sqrt{\pi}\left(1+\eta_{N}\right)$ with $\eta_{N}=O\left(1 / N^{2}\right)$. Now, since $\operatorname{Re} t>0$ (due to Result 1 ), we may write

$$
\begin{aligned}
\int_{t}^{1}\left(1-s^{2}\right)^{N} d s & =\int_{0}^{1-t^{2}} \omega^{N} \frac{d \omega}{2 \sqrt{1-\omega}} \\
& =\left(1-t^{2}\right)^{N+1} \int_{0}^{1} \lambda^{N} \frac{d \lambda}{2 \sqrt{1-\lambda\left(1-t^{2}\right)}}
\end{aligned}
$$

We write $\Omega=1-t^{2}$. If $|\Omega| \leq 1$ then we will prove that

$$
\inf _{\lambda \in[0,1]}|1-\lambda \Omega| \geq \frac{1}{2}|1-\Omega|
$$

This is obvious if $\operatorname{Re} \Omega \leq 0$ : we have $|1-\lambda \Omega| \geq 1$ and $|1-\Omega| \leq 2$. If $\operatorname{Re} \Omega>0, \Omega=\rho e^{i \varphi}(0<\rho \leq 1, \varphi \in(-\pi / 2, \pi / 2))$, we distinguish the case $\rho \leq \sin \varphi$ and $\rho>\sin \varphi$. If $\rho \leq \sin \varphi$, it is easily checked 
that $|1-\lambda \Omega| \geq|1-\Omega|$. If $\rho>\sin \varphi$, we have $|1-\lambda \Omega| \geq \sin \varphi$ and $|1-\Omega| \leq\left|1-e^{i \varphi}\right|=2|\sin (\varphi / 2)| ;$ hence

$$
|1-\lambda \Omega| \geq\left|\cos \frac{\varphi}{2}\right||1-\Omega| \geq \frac{\sqrt{2}}{2}|1-\Omega|
$$

Thus, we have for $\operatorname{Re} t>0$ and $\left|1-t^{2}\right| \leq 1$

$$
\left|\int_{t}^{1}\left(1-s^{2}\right)^{N} d s\right| \leq \frac{\left|1-t^{2}\right|^{N+1}}{N+1} \frac{1}{|t|} \leq \frac{1}{\sqrt{N}}\left(\frac{1}{\sqrt{N}|t|}\right) .
$$

If $|t \sqrt{N}| \geq 2 / \sqrt{\pi}$, we get

$$
\left|\sqrt{N} \int_{t}^{1}\left(1-s^{2}\right)^{N} d s\right| \leq \frac{1}{2} \sqrt{\pi},
$$

and thus $Q_{N}(t) \neq 0$ (for $N$ large enough so that $\left|\eta_{N}\right|<1 / 2$ ). If $\sqrt{N}|t| \leq 2 / \sqrt{\pi}$, then $t \sim-\bar{\gamma} / \sqrt{N}$ for a root $\gamma$ of $\operatorname{erfc}(z) \operatorname{such}$ that $|\gamma| \leq 2 / \sqrt{\pi}$; but the roots of $\operatorname{erfc}(z)$ satisfy $\pi / 2<|\operatorname{Arg} \gamma|<3 \pi / 4$ so that (for $N$ large enough) $|\operatorname{Arg} t|>\pi / 4$ and $t$ cannot lie inside the lemniscate $\left|1-t^{2}\right| \leq 1$.

We may now enter the core of our computations. We are going to give a precise description of $\int_{t}^{1}\left(1-s^{2}\right)^{N} d s$. Integration by parts gives us

$$
\begin{aligned}
\int_{t}^{1}\left(1-s^{2}\right)^{N} d s & =\frac{\left(1-t^{2}\right)^{N+1}}{2 t(N+1)}-\int_{t}^{1} \frac{\left(1-s^{2}\right)^{N+1}}{2 s^{2}(N+1)} d s \\
& =\frac{\left(1-t^{2}\right)^{N+1}}{2 t(N+1)}-\frac{\left(1-t^{2}\right)^{N+2}}{4(N+1)} \int_{0}^{1} \frac{\lambda^{N+1} d \lambda}{\left(1-\lambda\left(1-t^{2}\right)\right)^{3 / 2}} .
\end{aligned}
$$

We then define $\eta(t)$ as

$$
\eta(t)=\frac{\left|t^{2}\right|}{\inf _{\lambda \in[0,1]}\left|1-\lambda\left(1-t^{2}\right)\right|}
$$

We have

$$
\int_{t}^{1}\left(1-s^{2}\right)^{N} d s=\frac{\left(1-t^{2}\right)^{N+1}}{2 t(N+1)}\left(1+\frac{\left(1-t^{2}\right)}{2(N+2) t^{2}} \mu_{N}(t)\right)
$$


for $\operatorname{Re} t>0$ with

$$
\left|\mu_{N}(t)\right| \leq \eta(t)^{3 / 2}
$$

Of course, (35) is a good formula if $\mu_{N}(t)$ cannot explode. As a matter of fact, we will show that in the neighbourhood of the roots of $Q_{N}(s)$ we have $|\eta(t)| \leq C_{0}$ where $C_{0}$ doesn't depend on $N$ nor $t$; but we are still far from being able to prove it! The only obvious estimations on $\eta$ are the following ones: if $\operatorname{Re} t^{2} \geq 1$, we have of course $|\eta(t)|=\left|t^{2}\right|$, while if $\operatorname{Re} t^{2}<1$ and $\left|1-t^{2}\right|>1$ we have

$$
|\eta(t)|=\frac{\left|t^{2}\right|}{\left|\sin \left(\operatorname{Arg}\left(1-t^{2}\right)\right)\right|} .
$$

With help of formula (35) and a careful estimate of $\eta(t)$ in (36), we are going to prove:

Result 6. Let $\varphi_{N, k}=(8 k-1) \pi /(8 N+6)$. Then for $N$ large enough, the roots $x_{N, 1}, \ldots, x_{N, N}$ of $Q_{N}$ such that $x_{N, k} \neq-1$, ordered by

- for $1 \leq k \leq[(N+1) / 2]$, Re $x_{N, k} \geq 0$ and $x_{N, N+1-k}=\overline{x_{N, k}}$

- $\left|x_{N, 1}\right|<\left|x_{N, 2}\right|<\cdots<\left|x_{N,[(N+1) / 2]}\right|$

satisfy

$$
\begin{aligned}
& \mid x_{N, k}-\sqrt{2 \sin \varphi_{N, k}} e^{i\left(\pi / 4-\varphi_{N, k}\right) / 2} \\
& -\frac{e^{i\left(3 \pi / 4-3 \varphi_{N, k} / 2\right)}}{2 N \sqrt{2 \sin \varphi_{N, k}}} \log \left(2 \sqrt{2 N \pi \sin \varphi_{N, k}}\right) \mid
\end{aligned}
$$

$$
\leq C \frac{1}{\sqrt{N}} \max \left\{\frac{(1+\log k)^{2}}{k^{3 / 2}}, \frac{(1+\log N+1-k)^{2}}{(N+1-k)^{3 / 2}}\right\}
$$

where $C$ doesn't depend on $k$ nor $N$.

ProOF. Since $\varphi_{N, N+1-k}=\pi-\varphi_{N, k}$, it is enough to prove (37), for $1 \leq k \leq[(N+1) / 2]$, i.e. for the roots which lie in the upper half-plane. The proof is decomposed in the following steps: one first proves that $\operatorname{Arg}\left(1-x_{N, k}^{2}\right)$ cannot be too small, so that we have a first control on $\mu_{N}\left(x_{N, k}\right)$; then one gives through (35) a first estimate on $x_{N, k}$ and on the related error; this gives us a more precise information on $\operatorname{Arg}(1-$ $x_{N, k}^{2}$ ) and thus we may conclude with our final estimate. 
Step 1. We want to estimate $\operatorname{Arg}\left(1-x_{N, k}^{2}\right)$. We fix $\theta_{0} \in(\pi / 4, \pi / 2)$ so that the sector $\left\{z: \pi / 2 \leq|\operatorname{Arg} z| \leq \pi-\theta_{0}\right\}$ contains no zero of $\operatorname{erfc}(z)$ (remember that $\lim _{k \rightarrow+\infty} \operatorname{Arg} \gamma_{k}=3 \pi / 4$ ). We now distinguish the cases $\operatorname{Arg} x_{N, k} \in\left[0, \theta_{0}\right]$ and $\left.\operatorname{Arg} x_{N, k} \in\right] \theta_{0}, \pi / 2\left[\right.$. If $\operatorname{Re} 1-x_{N, k}^{2} \leq 0$, we know that $\eta\left(x_{N, k}\right) \leq\left|x_{N, k}\right|^{2} \leq 4$. If $\operatorname{Re} 1-x_{N, k}^{2}>0$ and $\operatorname{Arg} x_{N, k} \in$ $[0, \pi / 4]$, then we see that $\left|x_{N, k}\right|^{2} \leq\left|\tan \operatorname{Arg}\left(1-x_{N, k}^{2}\right)\right|$ (because $\omega=$ $1-x_{N, k}^{2}$ satisfies $\operatorname{Re} \omega \in(0,1]$ and $|\omega|>1$ so that $|\sin \operatorname{Arg} \omega| \leq \mid 1-$ $\omega|\leq| \tan \operatorname{Arg} \omega \mid)$; moreover we have $\left|x_{N, k}\right|^{2} \leq 4$; thus if $\mid \tan (\operatorname{Arg}(1-$ $\left.x_{N, k}^{2}\right) \mid \leq 4$, then we have

$$
\left|\sin \left(\operatorname{Arg}\left(1-x_{N, k}^{2}\right)\right)\right|=\frac{\mid \tan \left(\operatorname{Arg}\left(1-x_{N, k}^{2}\right) \mid\right.}{\sqrt{1+\tan ^{2}\left(\operatorname{Arg}\left(1-x_{N, k}^{2}\right)\right)}} \geq \frac{\left|x_{N, k}\right|^{2}}{\sqrt{17}}
$$

and $\eta\left(x_{N, k}\right) \leq \sqrt{17}$. On the other hand, if $\left|\tan \left(\operatorname{Arg}\left(1-x_{N, k}^{2}\right)\right)\right| \geq 4$, then we have $\left|\operatorname{Arg}\left(1-x_{N, k}^{2}\right)\right| \in[\operatorname{Arg} \tan 4, \pi / 2]$ and thus

$$
\left|\sin \left(\operatorname{Arg}\left(1-x_{N, k}^{2}\right)\right)\right| \geq \sin \operatorname{Arg} \tan 4=\frac{4}{\sqrt{17}} \geq \frac{\left|x_{N, k}\right|^{2}}{\sqrt{17}}
$$

and $\eta\left(x_{N, k}\right) \leq \sqrt{17}$ again.

If $\operatorname{Arg}\left(x_{N, k}\right) \in\left[\pi / 4, \theta_{0}\right]$, we have

$$
\left|\operatorname{Im}\left(1-x_{N, k}^{2}\right)\right|=\left|x_{N, k}^{2}\right|\left|\sin 2 \operatorname{Arg} x_{N, k}\right|
$$

so that

$$
\left|\operatorname{Im}\left(1-x_{N, k}^{2}\right)\right| \geq\left|x_{N, k}\right|^{2}\left|\sin 2 \theta_{0}\right|
$$

while

$$
\left|\sin \operatorname{Arg}\left(1-x_{N, k}^{2}\right)\right|=\frac{\left|\operatorname{Im}\left(1-x_{N, k}^{2}\right)\right|}{\left|1-x_{N, k}^{2}\right|} \geq \frac{1}{3}\left|\operatorname{Im}\left(1-x_{N, k}^{2}\right)\right|,
$$

so that

$$
\eta\left(x_{N, k}\right) \leq \frac{3}{\left|\sin 2 \theta_{0}\right|} .
$$

The difficult case is when $\theta_{0} \leq \operatorname{Arg} x_{N, k} \leq \pi / 2$ (as a matter of fact, we will see in step 3 that this case never occurs when $N$ is big enough!). For the moment, we will show that we have necessarily for such an $x_{N, k}$ (and provided $N$ is large enough) the inequality

$$
N\left|x_{N, k}\right|^{4} \geq \frac{\left|\cos \theta_{0}\right|}{100 C_{0}^{2}}=\varepsilon_{1},
$$


where $C_{0}$ is given by

$$
C_{0}=\max \left\{\sup _{|\sigma| \leq 1 / 2}\left|\frac{\sigma^{2}+\log \left(1-\sigma^{2}\right)}{\sigma^{4}}\right|, \sup _{|\sigma| \leq 1} \frac{\left|e^{\sigma}-1\right|}{|\sigma|}\right\} .
$$

Indeed, let $A_{0}>0$ be large enough so that for $A \geq A_{0}, e^{3 A^{2} \cos \left(2 \theta_{0}\right) / 4}$ $\left(1+A^{2} / 2\right)<1 / 100$ (remember that $\left.\cos 2 \theta_{0}<0\right), 4 /\left(A^{2}\left|\cos 2 \theta_{0}\right|\right)<$ $1 / 100$ and $A e^{A^{2} \cos \left(2 \theta_{0}\right) / 4}<1 / 100$. If $\sqrt{N}\left|x_{N, k}\right| \geq A_{0}$ and $N\left|x_{N, k}\right|^{4} \leq$ $\varepsilon_{1}$, we write

$$
Q_{N}\left(x_{N, k}\right)=\frac{1}{2}+\left(1+O\left(\frac{1}{N^{2}}\right)\right) \sqrt{\frac{N}{\pi}} \int_{0}^{x_{N, k}}\left(1-s^{2}\right)^{N} d s
$$

and thus

$$
\left|Q_{N}\left(x_{N, k}\right)\right| \geq \frac{1}{10} \sqrt{N}\left|\int_{0}^{x_{N, k}}\left(1-s^{2}\right)^{N} d s\right|-\frac{1}{2} .
$$

We write

$$
\left(1-s^{2}\right)^{N}=e^{-N s^{2}} e^{N\left(s^{2}-\log \left(1-s^{2}\right)\right)},
$$

since $|s| \leq \sqrt{\varepsilon_{1} / N} / 4$, we have $|s| \leq 1 / 2$ for $N$ large enough, thus

$$
\mid N\left(s^{2}-\log \left(1-s^{2}\right)\left|\leq C_{0}\right| N s^{4} \mid \leq \frac{1}{100},\right.
$$

thus

$$
\left|e^{N\left(s^{2}-\log \left(1-s^{2}\right)\right)}-1\right| \leq C_{0}^{2}\left|N s^{4}\right| .
$$

Thus, writing $x_{N, k}=\rho_{N, k} e^{i \theta_{N, k}}$, we get

$$
\begin{aligned}
\left|Q_{N}\left(x_{N, k}\right)\right| \geq & \frac{1}{10}\left|\int_{0}^{\sqrt{N} x_{N, k}} e^{-s^{2}} d s\right| \\
& -\frac{C_{0}^{2}}{10} \int_{0}^{\sqrt{N} \rho_{N, k}} e^{-s^{2} \cos 2 \theta_{N, k}} \frac{s^{4}}{N} d s-\frac{1}{2} \\
\geq & \frac{1}{10}\left|\int_{0}^{\sqrt{N} x_{N, k}} e^{-s^{2}} d s\right| \\
& -\frac{C_{0}^{2}}{10} \frac{\left(\sqrt{N} \rho_{N, k}\right)^{3}}{N\left|\cos 2 \theta_{N, k}\right|}
\end{aligned}
$$




$$
\begin{aligned}
& \int_{0}^{\sqrt{N} \rho_{N, k}} e^{-s^{2} \cos 2 \theta_{N, k}} s\left|\cos 2 \theta_{N, k}\right| d s-\frac{1}{2} \\
\geq & \frac{1}{10}\left|\int_{0}^{\sqrt{N} x_{N, k}} e^{-s^{2}} d s\right| \\
- & \frac{e^{-N \rho_{N, k}^{2} \cos 2 \theta_{N, k}}}{2 \sqrt{N} \rho_{N, k}}\left(\frac{C_{0}^{2}\left(\sqrt{N} \rho_{N, k}\right)^{4}}{10\left|\cos 2 \theta_{0}\right|}\right)-\frac{1}{2} .
\end{aligned}
$$

We have now to estimate $\int_{0}^{\sqrt{N} x_{N, k}} e^{-s^{2}} d s$. We write

$$
\begin{aligned}
& \int_{0}^{\sqrt{N} x_{N, k}} e^{-s^{2}} d s \\
& =e^{i \theta_{N, k}}\left(\int_{0}^{\sqrt{N} \rho_{N, k} / 2} e^{-s^{2} e^{2 i \theta} \theta_{N, k}} d s+\int_{\sqrt{N} \rho_{N, k} / 2}^{\sqrt{N} \rho_{N, k}} e^{-s^{2} e^{2 i \theta}, k} d s\right) \\
& =e^{i \theta_{N, k}}\left(I_{1}+I_{2}\right) .
\end{aligned}
$$

We have $\left|I_{1}\right| \leq e^{-N \rho_{N, k}^{2} \cos \left(2 \theta_{N, k}\right) / 4} \rho_{N, k} \sqrt{N} / 2$, while

$$
\begin{aligned}
I_{2} & =\left[\frac{e^{-s^{2} e^{2 i \theta_{N, k}}}}{-2 s e^{2 i \theta_{N, k}}}\right]_{\sqrt{N} \rho_{N, k} / 2}^{\sqrt{N} \rho_{N, k}}-\int_{\sqrt{N} \rho_{N, k} / 2}^{\sqrt{N} \rho_{N, k}} \frac{e^{-s^{2} e^{2 i \theta_{N, k}}}}{2 s^{2} e^{2 i \theta_{N, k}}} d s \\
& =\frac{e^{-N \rho_{N, k}^{2} e^{2 i \theta_{N, k}}}}{-2 \sqrt{N} \rho_{N, k} e^{2 i \theta_{N, k}}}-\frac{e^{-N \rho_{N, k}^{2} e^{2 i \theta_{N, k} / 4}}}{-\sqrt{N} \rho_{N, k} e^{2 i \theta_{N, k}}}-I_{3} .
\end{aligned}
$$

We have

$$
\begin{aligned}
\left|I_{3}\right| \leq & \frac{1}{4\left(\frac{1}{2} \sqrt{N} \rho_{N, k}\right)^{3}\left|\cos 2 \theta_{N, k}\right|} \\
& \cdot \int_{\sqrt{N} \rho_{N, k} / 2}^{\sqrt{N} \rho_{N, k}} e^{-s^{2} \cos 2 \theta_{N, k}} 2 s\left|\cos 2 \theta_{N, k}\right| d s \\
\leq & \frac{e^{-N \rho_{N, k}^{2} \cos 2 \theta_{N, k}}}{4\left(\frac{1}{2} \sqrt{N} \rho_{N, k}\right)^{3}\left|\cos 2 \theta_{0}\right|} .
\end{aligned}
$$


Thus we get

$$
\begin{aligned}
\left|Q_{N}\left(x_{N, k}\right)\right| & \\
\geq & \frac{1}{10} \frac{e^{-N \rho_{N, k}^{2} \cos 2 \theta_{N, k}}}{2 \sqrt{N} \rho_{N, k}} \\
& \cdot\left(1-2 e^{3 N \rho_{N, k}^{2} \cos 2 \theta_{N, k} / 4}-\frac{4}{N \rho_{N, k}^{2}\left|\cos 2 \theta_{0}\right|}\right. \\
& \quad-N \rho_{N, k}^{2} e^{3 N \rho_{N, k}^{2} \cos 2 \theta_{N, k} / 4}-\frac{C_{0}^{2} \varepsilon_{1}}{\left|\cos 2 \theta_{0}\right|} \\
& \left.\quad-10 \sqrt{N} \rho_{N, k} e^{N \rho_{N, k}^{2} \cos 2 \theta_{N, k}}\right) \\
\geq & \frac{1}{10} \frac{e^{-N \rho_{N, k}^{2} \cos 2 \theta_{N, k}}}{2 \sqrt{N} \rho_{N, k}}\left(1-\frac{2}{100}-\frac{1}{100}-\frac{1}{100}-\frac{1}{100}-\frac{10}{100}\right)>0
\end{aligned}
$$

which contradicts $Q_{N}\left(x_{N, k}\right)=0$. Up to now, we have proved that if $\arg x_{N, k}>\theta_{0}$ then either $\sqrt{N}\left|x_{N, k}\right| \leq A_{0}$ or $N\left|x_{N, k}\right|^{4} \geq \varepsilon_{1}$. But if $\left|x_{N, k}\right| \leq A_{0} / \sqrt{N}$ and $N$ is large enough, Result 4 ensures that $-\sqrt{N} x_{N, k}$ is close to a zero of $\operatorname{erfc}(z)$. This is not possible for $N$ large enough since the distance between $\left\{z: \pi / 2 \leq|\operatorname{Arg} z| \leq \pi-\theta_{0}\right\}$ and $\{z: \operatorname{erfc}(z)=0\}$ is positive.

Thus we must have $N\left|x_{N, k}\right|^{4} \geq \varepsilon_{1}$. Write again $x_{N, k}=\rho_{N, k} e^{i \theta_{N, k}}$; since $\left|x_{N, k}-1\right| \leq 1$ by Result 1 , we have $\rho_{N, k} \leq 2 \cos \theta_{N, k}$; thus $2 \cos \theta_{N, k} \geq\left(\varepsilon_{1} / N\right)^{1 / 4}$ and

$$
\left|\operatorname{Im} x_{N, k}^{2}\right|=\left|x_{N, k}^{2}\right|\left|\sin 2 \theta_{N, k}\right| \geq \sin \theta_{0}\left(\frac{\varepsilon_{1}}{N}\right)^{1 / 4}\left|x_{N, k}\right|^{2}
$$

We thus have proved

$$
\eta\left(x_{N, k}\right)=\frac{\left|x_{N, k}\right|^{2}\left|1-x_{N, k}^{2}\right|}{\left|\operatorname{Im} x_{N, k}^{2}\right|} \leq \frac{3 N^{1 / 4}}{\left(\sin \theta_{0}\right) \varepsilon_{1}^{1 / 4}}=C_{1}^{N^{1 / 4}} .
$$

We thus have proved

- if $\operatorname{Arg} x_{N, k}<\theta_{0}$,

$$
\left|\mu_{N}\left(x_{N, k}\right)\right| \leq \eta\left(x_{N, k}\right)^{3 / 2} \leq C_{2},
$$


- if $\operatorname{Arg} x_{N, k}>\theta_{0}$,

$$
\begin{aligned}
\left|\mu_{N}\left(x_{N, k}\right)\right| & \leq \eta\left(x_{N, k}\right)^{3 / 2} \\
& \leq\left(C_{1} N^{1 / 4}\right)^{3 / 2} \\
& =C_{1}^{3 / 2} \frac{\left(N \mid x_{N, k}^{2}\right)^{3 / 4}}{\left(N\left(\left|x_{N, k}\right|^{4}\right)^{3 / 8}\right.} \\
& \leq \frac{C_{1}^{3 / 2}}{\varepsilon_{1}^{3 / 8}}\left(N\left|x_{N, k}\right|^{2}\right)^{3 / 4} .
\end{aligned}
$$

In any case, we have

$$
\left|\mu_{N}\left(x_{N, k}\right)\right| \leq C\left(N\left|x_{N, k}\right|^{2}\right)^{3 / 4} .
$$

(Remember that $\lim _{N \rightarrow+\infty} \inf _{k} N\left|x_{N, k}\right|^{2}=\left|\gamma_{1}\right|^{2}>0$ ).

Step 2. We are now able to give an estimate for $x_{N, k}$. Let us consider a root $y \neq-1$ of $Q_{N}$ such that $\operatorname{Im} y \geq 0$. We have

$$
\int_{y}^{1}\left(1-s^{2}\right)^{N} d s=2 \frac{4^{N}(N !)^{2}}{(2 N+1) !}
$$

hence from (35) and (36),

$$
\frac{\left(1-y^{2}\right)^{N+1}}{2(N+1) \sqrt{\pi} y}\left(1+O\left(\frac{\eta(y)^{3 / 2}}{N|y|^{2}}\right)\right)=\sqrt{\frac{\pi}{N}}\left(1+O\left(\frac{1}{N^{2}}\right)\right)
$$

(where $\alpha=O(\varepsilon(N, y)$ ) means that $|\alpha| / \varepsilon(N, y) \leq C$ for a positive constant $C$ which doesn't depend neither on $N$ nor on $y$ ). Taking the $(N+1)$-th root of the modulus of both terms of equality (39), we get

$$
\begin{aligned}
\left|1-y^{2}\right|= & 1+\frac{1}{N+1} \log \left(2 \sqrt{N \pi} \frac{N+1}{N}|y|\right) \\
& +O\left(\frac{(\log N)^{2}}{N^{2}}\right)+O\left(\frac{1}{N^{3}}\right)+O\left(\frac{\eta(y)^{3 / 2}}{N^{2}|y|^{2}}\right) \\
= & 1+\frac{1}{N} \log (2 \sqrt{N \pi}|y|)+O\left(\frac{(\log N)^{2}}{N^{2}}\right)+O\left(\frac{\eta(y)^{3 / 2}}{N^{2}|y|^{2}}\right) .
\end{aligned}
$$


Now, we write $1-y^{2}=\rho e^{-i \varphi}(\varphi \in[0, \pi], \rho>0)$, so that $y=$ $\sqrt{1-\rho e^{-i \varphi}}$. We have found

$$
\begin{aligned}
|1-\rho| & =O\left(\frac{1}{N} \log (\sqrt{N}|y|)\right)+O\left(\frac{(\log N)^{2}}{N^{2}}\right)+O\left(\frac{\eta(y)^{3 / 2}}{N^{2}|y|^{2}}\right) \\
& =O\left(\frac{1}{N} \log (\sqrt{N}|y|)\right)
\end{aligned}
$$

(since $1 / C N \leq \log (\sqrt{N}|y|) / N \leq C \log N / N$, while $\eta(y)^{3 / 2} /\left(N^{2}|y|^{2}\right)$ $\left.\leq C /\left(N\left(N|y|^{2}\right)\right) \leq C^{\prime} / N\right)$. Thus $1-\rho e^{-i \varphi}=1-e^{-i \varphi}+(1-\rho) e^{-i \varphi}$ with

$$
\left|\frac{(1-\rho) e^{-i \varphi}}{1-\rho e^{-i \varphi}}\right|=O\left(\frac{\log (\sqrt{N}|y|)}{N|y|^{2}}\right)
$$

and we find

$$
\begin{aligned}
y & =\sqrt{\left(1-e^{-i \varphi}\right)\left(1+O\left(\frac{\log \sqrt{N}|y|}{N|y|^{2}}\right)\right)} \\
& =\sqrt{2 \sin \left(\frac{\varphi}{2}\right)} e^{i(\pi / 4-\varphi / 4)}\left(1+O\left(\frac{\log \sqrt{N}|y|}{N|y|^{2}}\right)\right) .
\end{aligned}
$$

We insert this result in (39) and take the phase

$$
-(N+1) \varphi-\frac{\pi}{4}+\frac{\varphi}{4}+O\left(\frac{\log \sqrt{N}|y|}{N|y|^{2}}\right)+O\left(\frac{\eta(y)^{3 / 2}}{N|y|^{2}}\right)=-2 k \pi
$$

or

$$
\varphi=\frac{8 k-1}{4 N+3} \pi+O\left(\frac{\log \sqrt{N}|y|}{N^{2}|y|^{2}}\right)+O\left(\frac{\eta(y)^{3 / 2}}{N^{2}|y|^{2}}\right) .
$$

If we assume $\sqrt{N}|y| \geq A_{0}$ where $A_{0}$ is big enough so that

$$
O\left(\frac{\log A_{0}}{N A_{0}^{2}}\right)+O\left(\frac{1}{N A_{0}^{1 / 2}}\right)
$$

is less than $4 \pi /(4 N+3)$ ( $A_{0}$ being chosen independently from $\left.N\right)$, we see that $0 \leq \varphi \leq \pi$ implies $0 \leq k \leq[(N+1) / 2]$; moreover since

$$
|y|=\sqrt{2 \sin \left(\frac{\varphi}{2}\right)}\left(1+O\left(\frac{\log \sqrt{N}|y|}{N|y|^{2}}\right)\right)
$$


we must have

$$
2 \sin \left(\frac{\varphi}{2}\right) \geq \frac{A_{0}^{2}}{N}+O\left(\frac{\log \sqrt{N}|y|}{N^{2}|y|^{2}}\right) .
$$

We take $A_{0}^{2}=\sqrt{2 K_{0} \pi}$, where $K_{0}$ is big enough; we then see that we must have $k>K_{0}$.

If $\sqrt{N}|y| \leq \sqrt{2 K_{0} \pi}$, we know that (provided $N$ is big enough) $y \sim-\bar{\gamma}_{k} / \sqrt{N}$ for $k \in\left\{1, \ldots, K_{0}\right\}$. We have moreover found candidates $y_{N, k}$ for the remaining roots $x_{N, k}, K_{0}<k \leq[(N+1) / 2]$, which are given by

$$
1-y_{N, k}^{2}=\left(1+\frac{1}{N} \log 2 \sqrt{2 N \pi \sin \varphi_{N, k}}\right) e^{-2 i \varphi_{N, k}},
$$

for $K_{0}<k \leq[(N+1) / 2]$ and $\varphi_{N, k}=(8 k-1) \pi /(8 N+6)$.

More precisely, we have shown that if $Q_{N}(y)=0, \operatorname{Im} y \geq 0, y \neq-1$ and $\sqrt{N}|y| \geq \sqrt{2 K_{0} \pi}$, then for some $k \in\left\{K_{0}+1, \ldots,[(N+1) / 2]\right\}$ we have

$$
1-y^{2}=1-y_{N, k}^{2}+O\left(\frac{(\log N)^{2}}{N^{2}}\right)+O\left(\frac{\eta(y)^{3 / 2}}{N^{2}|y|^{2}}\right)+O\left(\frac{\log \sqrt{N}|y|}{N^{2}|y|^{2}}\right) .
$$

We are going now to prove that, provided that $K_{0}$ is fixed large enough (and provided thereafter that $N$ is large enough), for each $y_{N, k}$ there is exactly one root $y$ satisfying (42). Notice that $\left|y_{N, k}^{2}-y_{N, k+1}^{2}\right| \geq C_{0} / N$ while

$$
\begin{aligned}
O\left(\frac{(\log N)^{2}}{N^{2}}\right)+O\left(\frac{\eta(y)^{3 / 2}}{N^{2}|y|^{2}}\right) & +O\left(\frac{\log \sqrt{N}|y|}{N^{2}|y|^{2}}\right) \\
& \leq C \frac{1}{N}\left(\frac{(\log N)^{2}}{N}+\frac{1}{(\sqrt{N}|y|)^{1 / 2}}\right)
\end{aligned}
$$

Indeed, let's write $s=\sqrt{y_{N, k}^{2}-v}$ where $|v|=\eta_{0} / N, \eta_{0}$ small enough. We are going to estimate $Q_{N}(s)$. We know that

$$
\int_{s}^{1}\left(1-\sigma^{2}\right)^{N} d \sigma=\frac{\left(1-s^{2}\right)^{N+1}}{2 s(N+1)}\left(1+O\left(\frac{\eta(s)}{N|s|^{2}}\right)\right)
$$

where $\eta(s)$ is bounded independently of $s$ provided that $|1-s|<1$, $\left|1-s^{2}\right|>1$ and $|\operatorname{Arg} s|<\theta_{0}$ (where $\theta_{0} \in(\pi / 4, \pi / 2)$ ). Thus, we are 
going to estimate $|1-s|,\left|1-s^{2}\right|$ and $|\operatorname{Arg} s|$. We have obviously from (41)

$$
y_{N, k}^{2}=1-e^{-2 i \varphi_{N, k}}+O\left(\frac{\log k}{N}\right)=\left(1-e^{-2 i \varphi_{N, k}}\right)\left(1+O\left(\frac{\log k}{k}\right)\right)
$$

and such an estimate holds as well for $s^{2}$. (We see also from (41) that

$$
\begin{aligned}
\left|1-s^{2}\right| & \geq 1+\frac{1}{N} \log 2 \sqrt{2 N \pi \sin \varphi_{N, k}}-\frac{\eta_{0}}{N} \\
& \geq 1+\frac{1}{N} \log 2 \sqrt{4 \pi K_{0}}-\frac{\eta_{0}}{N} \\
& >1
\end{aligned}
$$

provided $\eta_{0}$ is small enough). Thus we find that

$$
\operatorname{Arg} s^{2}=\frac{\pi}{2}-\varphi_{N, k}+O\left(\frac{\log k}{k}\right)<2 \theta_{0}
$$

if $K_{0}$ is large enough (so that $\left.O\left(\log K_{0} / K_{0}\right)<2 \theta_{0}-\pi / 2\right)$ and thus

$$
\operatorname{Arg} s=\frac{\pi}{4}-\frac{1}{2} \varphi_{N, k}+O\left(\frac{\log k}{k}\right) \in\left(-\theta_{0}, \theta_{0}\right) .
$$

Moreover,

$$
|s|=\sqrt{2 \sin \varphi_{N, k}}\left(1+O\left(\frac{\log k}{k}\right)\right)
$$

and this latter estimate gives $|s|<2 \cos (\operatorname{Arg} s)$ : if $\varphi_{N, k}>\varepsilon_{0}$ (where $\varepsilon_{0}$ is fixed small enough as we shall see below) and $K_{0}$ and $N$ are large enough we have

$$
\sqrt{2 \sin \varphi_{N, k}}\left(1+O\left(\frac{\log k}{k}\right)\right) \leq \sqrt{2}\left(1+C \frac{\log K_{0}}{K_{0}}\right) \leq \sqrt{2}\left(1+\frac{\varepsilon_{0}}{100}\right)
$$

while

$$
\begin{aligned}
2 \cos (\operatorname{Arg} s) & \geq 2 \cos \left(\frac{\pi}{4}-C \frac{\log K_{0}}{K_{0}}\right) \\
& \geq 2 \cos \left(\frac{\pi}{4}-\frac{\varepsilon_{0}}{3}\right) \\
& \geq \sqrt{2}\left(1+\frac{2 \varepsilon_{0}}{3 \pi}-\frac{\varepsilon_{0}^{2}}{2}\right)
\end{aligned}
$$


On the other hand, if $\varphi_{N, k}<\varepsilon_{0}$ we find

$$
\sqrt{2 \sin \varphi_{N, k}}\left(1+O\left(\frac{\log k}{k}\right)\right) \leq \sqrt{2 \varepsilon_{0}} \sqrt{1+C \frac{\log K_{0}}{K_{0}}} \leq C^{\prime} \sqrt{\varepsilon_{0}},
$$

while $2 \cos (\operatorname{Arg} s) \geq 2 \cos \theta_{0}$; thus if $\varepsilon_{0}$ is small enough to ensure $\varepsilon_{0}<$ $4 /(3 \pi)-1 / 50$ and $\varepsilon_{0}<4 \cos ^{2} \theta_{0} / C^{2}$ we find $|s|<2 \cos (\operatorname{Arg} s)$. But this latter inequality is equivalent to $|1-s|<1$. Thus we found

$$
Q_{N}(s)=1-\left(1+O\left(\frac{1}{N^{2}}\right)\right) \sqrt{\frac{N}{\pi}} \frac{\left(1-s^{2}\right)^{N+1}}{2 s(N+1)}\left(1+O\left(\frac{1}{\left|N s^{2}\right|}\right)\right) .
$$

We have moreover:

$$
\begin{gathered}
\left(1-s^{2}\right)^{N+1}=\left(1-y_{N, k}^{2}\right)^{N+1}\left(1+\frac{v}{1-y_{N, k}^{2}}\right)^{N+1} \\
=\left(1-y_{N, k}^{2}\right)^{N+1}\left(1+\frac{N v}{1-y_{N, k}^{2}}+O\left(N^{2} v^{2}\right)\right) \\
s=\sqrt{y_{N, k}^{2}-v}=y_{N, k}\left(1-\frac{v}{2 y_{N, k}^{2}}+O\left(\frac{v^{2}}{y_{N, k}^{4}}\right)\right) .
\end{gathered}
$$

This gives, since $|s|$ has $\sqrt{k / N}$ as order of magnitude

$$
\begin{aligned}
Q_{N}(s)=1- & \left(1+O\left(\frac{1}{k}\right)\right) \frac{\left(1-y_{N, k}^{2}\right)^{N+1}}{2 \sqrt{N \pi} y_{N, k}} \\
& \cdot\left(1+\frac{N v}{1-y_{N, k}^{2}}+\frac{v}{2 y_{N, k}^{2}}+O\left(N^{2} v^{2}\right)+O\left(\frac{v^{2}}{y_{N, k}^{4}}\right)\right) .
\end{aligned}
$$

Moreover

$$
\left|y_{N, k}\right| \geq 2 \sqrt{\frac{8 k-1}{8 N+6}}\left(1+O\left(\frac{1}{k} \log k\right)\right)
$$

and

$$
y_{N, k}=\sqrt{2 \sin \left(\frac{8 k-1}{8 N+6} \pi\right)} e^{i(\pi / 4-(8 k-1) \pi /(16 N+12))}\left(1+O\left(\frac{1}{k} \log k\right)\right),
$$


so that

$$
\begin{aligned}
& \frac{\left(1-y_{N, k}^{2}\right)^{N+1}}{2 \sqrt{N \pi} y_{N, k}} \\
& =\frac{\left(1+\frac{1}{N} \log 2 \sqrt{2 N \pi \sin \left(\frac{8 k-1}{8 N+6} \pi\right)}\right)^{N}}{2 \sqrt{2 N \pi \sin \left(\frac{8 k-1}{8 N+6} \pi\right)}\left(1+O\left(\frac{1}{k} \log k\right)\right)} \\
& =\left(1+O\left(\frac{1}{N^{2}}(\log k)^{2}\right)\right)^{N}\left(1+O\left(\frac{1}{k} \log k\right)\right)
\end{aligned}
$$

and finally

$$
\begin{aligned}
& Q_{N}(s)=1-\left(1+O\left(\frac{1}{k}(\log k)^{2}\right)\right) \\
& \cdot\left(1+\frac{N v}{1-y_{N, k}^{2}}+\frac{v}{2 y_{N, k}^{2}}+O\left(N^{2} v^{2}\right)+O\left(\frac{v^{2}}{y_{N, k}^{4}}\right)\right) .
\end{aligned}
$$

Now, we write

$$
R_{N, k}(s)=N \frac{v}{1-y_{N, k}^{2}}=N \frac{y_{N, k}^{2}-s^{2}}{1-y_{N, k}^{2}} .
$$

Since $|v|=\eta_{0} / N$, we have

$$
\left|R_{N, k}(s)\right|=\eta_{0}\left(1+O\left(\frac{\log k}{N}\right)\right)
$$

while

$$
\left|Q_{N}(s)-R_{N, k}(s)\right|=O\left(\frac{(\log k)^{2}}{k}\right)+O\left(\frac{\eta_{0}}{k}\right)+O\left(\eta_{0}^{2}\right)
$$

We choose $\eta_{0}$ small enough to ensure that the $O\left(\eta_{0}^{2}\right)$ term is smaller than $\eta_{0} / 2$ (independently of $N$ and $k$ ), and then choose $K_{0}$ large enough to ensure that $O\left((\log k)^{2} / k\right)+O\left(\eta_{0} / k\right)$ is smaller than $\eta_{0} / 4$ for $k>K_{0}$. For this choice of $K_{0}$, we get

$$
\left|Q_{N}(s)-R_{N, k}(s)\right|<\frac{3}{4} \eta_{0}<\left|R_{N, k}(s)\right|
$$


Thus, by Rouché's theorem, $Q_{N}(s)$ and $R_{N, k}(s)$ have the same number of roots inside the domain $\left\{\left|y_{N, k}^{2}-s^{2}\right| \leq \eta_{0} / N\right.$, Re $\left.s>0\right\}$.

Step 3. We have thus found a number $K_{0}$ so that for $N$ large enough we may list the roots $x_{N, 1}, \ldots, x_{N,[(N+1) / 2]}$ of $Q_{N}$ with $x_{N, k} \neq-1$, $\operatorname{Im} x_{N, k} \geq 0,\left|x_{N, k}\right|<\left|x_{N, k+1}\right|$ in the following way:

- for $k \leq K_{0},\left|x_{N, k}\right|<\sqrt{2 K_{0} \pi / N}$ and $x_{N, k} \sim-\bar{\gamma}_{k} / \sqrt{N}$,

- for $k \geq K_{0}$,

$$
\left|x_{N, k}^{2}-y_{N, k}^{2}\right|=O\left(\frac{\eta\left(x_{N, k}\right)^{3 / 2}}{N^{2}\left|x_{N, k}\right|^{2}}\right)+O\left(\frac{\log \left(\sqrt{N}\left|x_{N, k}\right|\right)}{N^{2}\left|x_{N, k}\right|^{2}}\right)
$$

where $y_{N, k}$ is given by (41).

Moreover, we have seen in step 2 that in that case we must have $\operatorname{Arg} x_{N, k}<\theta_{0}$, hence $\eta\left(x_{N, k}\right)$ is bounded independently of $N$ and $k$. Moreover $x_{N, k}$ is of order of magnituge $\sqrt{k / N}$, hence

$$
\left|x_{N, k}^{2}-y_{N, k}^{2}\right|=O\left(\frac{\log k}{N k}\right)
$$

Thus we find

$$
\begin{aligned}
1-x_{N, k}^{2}= & \left(1+\frac{1}{N} \log 2 \sqrt{2 N \pi \sin \left(\frac{8 k-1}{8 N+6} \pi\right)}\right) \\
& \cdot e^{-2 i \pi(8 k-1) /(8 N+6)}+O\left(\frac{\log k}{N k}\right)
\end{aligned}
$$

and thus

$$
\begin{aligned}
x_{N, k}^{2}= & \left(1-e^{-2 i \pi(8 k-1) /(8 N+6)}\right) \\
& \cdot\left(1-\frac{e^{-2 i \pi(8 k-1) /(8 N+6)}}{N\left(1-e^{-2 i \pi(8 k-1) /(8 N+6)}\right)} \log 2 \sqrt{2 N \pi \sin \left(\frac{8 k-1}{8 N+6} \pi\right)}\right. \\
& \left.+O\left(\frac{\log k}{k^{2}}\right)\right)
\end{aligned}
$$


which gives

$$
x_{N, k}=e^{i(\pi / 4-(8 k-1) \pi /(16 N+12)} \sqrt{2 \sin \left(\frac{8 k-1}{8 N+6} \pi\right)}
$$

$$
\begin{aligned}
& \cdot\left(1+\frac{e^{i(\pi / 2-(8 k-1) \pi /(8 N+6))}}{4 N \sin \left(\frac{8 k-1}{8 N+6} \pi\right)} \log 2 \sqrt{2 N \pi \sin \left(\frac{8 k-1}{8 N+6} \pi\right)}\right. \\
& \left.+O\left(\frac{\log k}{k^{2}}\right)\right)
\end{aligned}
$$

which gives (37) for $k>K_{0}$. For $k \leq K_{0}$, (37) says only that $x_{N, k}$ is $O(1 / \sqrt{N})$, which we already known since $\sqrt{N}\left|x_{N, k}\right| \leq \sqrt{2 K_{0} \pi}$.

Thus we have proved Result 6 .

A nice corollary of Result 6 is that we may recover formula (33) on the roots of $\operatorname{erfc}(z)$ :

Corollary. The $k$-th root $\gamma_{k}$ of $\operatorname{erfc}(z)$ such that $\operatorname{Im} \gamma_{k}>0$ is given by

$$
\gamma_{k}=e^{3 i \pi / 4} \sqrt{\left(2 k-\frac{1}{4}\right) \pi}
$$

$$
\cdot\left(1-\frac{i}{2\left(2 k-\frac{1}{4}\right) \pi} \log 2 \sqrt{\pi} \sqrt{\left(2 k-\frac{1}{4}\right) \pi}+O\left(\frac{(\log k)^{2}}{k^{2}}\right)\right)
$$

Proof. It is enough to use formula (37) for $x_{N, k}$ with $N, k \rightarrow+\infty$ and $k<\log N / 8$ : we have

$$
x_{N, k}=-\frac{-\bar{\gamma}_{k}}{\sqrt{N}}+O\left(\frac{1}{N}\right) \quad \text { and } \quad \frac{k}{N}=O\left(\frac{\log N}{N}\right),
$$

thus we find $\gamma_{k}$. The only thing to check is the exact number of roots $\gamma$ such that $|\gamma| \leq \sqrt{2 K_{0} \pi}$ (since we used formula (33) to give it). But this is an old and classical result of Nevanlinna [9], and thus we may recover formula (33) from formula (37). 


\section{Big roots of $Q_{N}$ : further estimates.}

Though Result 6 is enough for the proof of theorems 1 to 3 (provided we improve result $\mathrm{n}^{\circ} 4$ for the smaller roots), we may give even more precise estimations for the roots $x_{N, k}$. For instance, we may integrate by parts one step further formula (35) and thus get an $O\left((\log k)^{3}\right.$ $\left./ N k^{2}\right)$ error instead of $O(\log k / N k)$ for $1-x_{N, k}^{2}$.

More generally, how far can we compute $\int_{t}^{1}\left(1-s^{2}\right)^{N} d s$ ? We have

$$
\int_{t}^{1}\left(1-s^{2}\right)^{N} d s=\left(1-t^{2}\right)^{N+1} \int_{0}^{1} \lambda^{N} \frac{d \lambda}{2 \sqrt{1-\lambda\left(1-t^{2}\right)}} .
$$

If we write

$$
1-\lambda\left(1-t^{2}\right)=t^{2}\left(1+\frac{1-t^{2}}{t^{2}}(1-\lambda)\right),
$$

we see that if $\operatorname{Re} t^{2}>1 / 2$ (so that $\left|1-t^{2}\right|<t^{2}$ ), we may develop $\left(\sqrt{1-\lambda\left(1-t^{2}\right)}\right)^{-1}$ as a Taylor series in $(1-\lambda)$ and find (for $\operatorname{Re} t^{2}>$ $1 / 2)$

$$
\frac{1}{\sqrt{1-\lambda\left(1-t^{2}\right)}}=\frac{1}{t} \sum_{k=0}^{+\infty}(-1)^{k} \frac{2 k !}{4^{k}(k !)^{2}}\left(\frac{(1-\lambda)\left(1-t^{2}\right)}{t^{2}}\right)^{k}
$$

which gives

$$
\left\{\begin{array}{l}
\text { for } \operatorname{Re} t>0 \text { and } \operatorname{Re} t^{2}>\frac{1}{2} \\
\int_{t}^{1}\left(1-s^{2}\right)^{N} d s \\
\quad=\frac{\left(1-t^{2}\right)^{N+1}}{2 t} \sum_{k=0}^{+\infty}(-1)^{k} \frac{(2 k) !}{4^{k}(k !)^{2}} \frac{N ! k !}{(N+k+1) !}\left(\frac{1-t^{2}}{t^{2}}\right)^{k} .
\end{array}\right.
$$

Unfortunately, we are mostly interested in small $t$ 's (remember that $\left.x_{N, k}=O(\sqrt{k / N})\right)$. (46) has to be replaced by an asymptotic formula (which is obtained by repeatedly integrating by parts)

$$
\left\{\begin{array}{l}
\text { for } \operatorname{Re} t>0 \text { and } M \in \mathbb{N} \\
\qquad \begin{array}{l}
\int_{t}^{1}\left(1-s^{2}\right)^{N} d s \\
\quad=\frac{\left(1-t^{2}\right)^{N+1}}{2 t} \sum_{k=0}^{M}(-1)^{k} \frac{(2 k) !}{4^{k}(k !)^{2}} \frac{N ! k !}{(N+k+1) !}\left(\frac{1-t^{2}}{t^{2}}\right)^{k} \\
\quad+R_{M, N}(t)
\end{array}
\end{array}\right.
$$


where the remainder

$$
\begin{aligned}
R_{M, N}(t)= & (-1)^{M+1}\left(1-t^{2}\right)^{N+M+2} \frac{(2 M+2) !}{4^{M+1}((M+1) !)^{2}} \\
& \cdot \frac{N !(M+1) !}{(N+M+2) !} \int_{0}^{1} \frac{\lambda^{N+M+1} d \lambda}{\left(1-\lambda\left(1-t^{2}\right)\right)^{1 / 2+M+1}}
\end{aligned}
$$

may be estimated by

$$
\begin{aligned}
\left|R_{M, N}(t)\right| \leq & \left|\frac{\left(1-t^{2}\right)^{N+1}}{2 t}\right| \frac{(2 M+2) !}{4^{M+1}((M+1) !)^{2}} \frac{(M+1) ! N !}{(N+M+2) !} \\
& \cdot\left|\frac{1-t^{2}}{t^{2}}\right|^{M+1} \eta(t)^{1 / 2+M+1} .
\end{aligned}
$$

$M=0$ gave Result $6 . M=1$ gives the following result:

Result 7. Writing $\varphi_{N, k}=(8 k-1) \pi /(8 N+6)$ and

$$
\lambda_{k}=\log 2 \sqrt{2 N \pi \sin \varphi_{N, k}},
$$

we have more precisely for all $k \in\{1, \ldots, N\}$

$1-x_{N, k}^{2}=e^{-2 i \varphi_{N, k}}$

$$
\begin{aligned}
& \cdot\left(1+\frac{1}{N} \lambda_{k}+\frac{1}{N^{2}}+\frac{\lambda_{k}}{N^{2}}+\frac{\lambda_{k}^{2}}{2 N^{2}}+\frac{i e^{-i \varphi_{N, k}}}{4 N^{2} \sin \varphi_{N, k}}\left(\lambda_{k}-1\right)\right) \\
& +\varepsilon_{N, k}
\end{aligned}
$$

where

$$
\left|\varepsilon_{N, k}\right| \leq C \max \left\{\frac{1+(\log k)^{3}}{N k^{2}}, \frac{1+\log (N+1-k)^{3}}{N(N+1-k)^{2}}\right\}
$$

and $C$ doesn't depend neither on $N$ nor on $K$.

Proof. We assume $k \leq[(N+1) / 2]$. We write $1-x_{N, k}^{2}=1-y_{N, k}^{2}+v$ and the problem is to estimate $v$. We already know $v=O(\log k /(N k))$. Furthermore, we know that

$$
\int_{x_{N, k}}^{1}\left(1-s^{2}\right)^{N} d s=\frac{24^{N}(N !)^{2}}{(2 N+1) !}=\sqrt{\frac{\pi}{N}}\left(1+O\left(\frac{1}{N^{2}}\right)\right)
$$


and

$$
\int_{x_{N, k}}^{1}\left(1-s^{2}\right)^{N} d s=\frac{\left(1-x_{N, k}^{2}\right)^{N+1}}{2(N+1) x_{N, k}}\left(1-\frac{1-x_{N, k}^{2}}{2(N+2) x_{N, k}^{2}}+O\left(\frac{1}{N^{2} x_{N, k}^{4}}\right)\right) .
$$

Now, write

$$
\begin{aligned}
\frac{1-x_{N, k}^{2}}{2(N+2) x_{N, k}^{2}} & =\frac{1-y_{N, k}^{2}}{2(N+2) y_{N, k}^{2}}+O\left(\frac{v}{k}\right)+O\left(\frac{N v}{k^{2}}\right) \\
& =\frac{1-y_{N, k}^{2}}{2(N+2) y_{N, k}^{2}}+O\left(\frac{\log k}{k^{3}}\right)
\end{aligned}
$$

and

$$
\begin{aligned}
\frac{1-y_{N, k}^{2}}{2(N+2) y_{N, k}^{2}} & =\frac{e^{-2 i \varphi_{N, k}}}{2(N+2) y_{N, k}^{2}}+O\left(\frac{\log k}{N k}\right) \\
& =\frac{e^{-2 i \varphi_{N, k}}}{2 N\left(1-e^{-2 i \varphi_{N, k}}\right)}+O\left(\frac{\log k}{k^{2}}\right),
\end{aligned}
$$

so that

$$
1-\frac{1-x_{N, k}^{2}}{2(N+2) x_{N, k}^{2}}+O\left(\frac{1}{N^{2} x_{N, k}^{4}}\right)=1+\frac{i e^{-i \varphi_{N, k}}}{4 N \sin \varphi_{N, k}}+O\left(\frac{\log k}{k^{2}}\right) .
$$

We now turn our attention to $\left(1-x_{N, k}^{2}\right)^{N+1} /\left(2(N+1) x_{N, k}\right)$. We have

$$
\begin{aligned}
& 2(N+1) x_{N, k} \sqrt{\frac{\pi}{N}} \\
&=2\left(1+\frac{1}{N}\right) \sqrt{N \pi} \sqrt{y_{N, k}^{2}-v} \\
&=2\left(1+\frac{1}{N}\right) \sqrt{N \pi} \sqrt{1-e^{-2 i \varphi_{N, k}}-\frac{e^{-2 i \varphi_{N, k}}}{N} \lambda_{N, k}+O\left(\frac{\log k}{N k}\right)} \\
&=2\left(1+\frac{1}{N}\right) \sqrt{N \pi} \sqrt{2 \sin \varphi_{N, k}} e^{i\left(\pi / 4-\varphi_{N, k} / 2\right)} \\
& \cdot\left(1+\frac{i e^{-i \varphi_{N, k}}}{4 N \sin \varphi_{N, k}} \lambda_{N, k}+O\left(\frac{(\log k)^{2}}{k^{2}}\right)\right)
\end{aligned}
$$


and

$$
\begin{aligned}
\left(1-x_{N, k}^{2}\right)^{N+1} & =\left(1-y_{N, k}^{2}\right)^{N+1}\left(1+\frac{v}{1-y_{N, k}^{2}}\right)^{N+1} \\
& =\left(1-y_{N, k}^{2}\right)^{N+1}\left(1+\frac{(N+1) v}{1-y_{N, k}^{2}}+O\left(\frac{(\log k)^{2}}{k^{2}}\right)\right) \\
& =\left(1-y_{N, k}^{2}\right)^{N+1}\left(1+N v e^{2 i \varphi_{N, k}}+O\left(\frac{(\log k)^{2}}{k^{2}}\right)\right) .
\end{aligned}
$$

Finally we have

$$
\begin{aligned}
& \frac{\left(1-y_{N, k}^{2}\right)^{N+1}}{2 \sqrt{2 N \pi \sin \varphi_{N, k}} e^{i\left(\pi / 4-\varphi_{N, k} / 2\right)}} \\
& =\left(\frac{1+\frac{1}{N} \lambda_{N, k}}{1+\frac{1}{N+1} \lambda_{N, k}+\frac{1}{2(N+1)^{2}} \lambda_{N, k}^{2}+O\left(\frac{(\log k)^{3}}{N^{3}}\right)}\right)^{N+1} \\
& =1-\frac{1}{N} \lambda_{N, k}-\frac{1}{2 N} \lambda_{N, k}^{2}+O\left(\frac{(\log k)^{3}}{N^{2}}\right)
\end{aligned}
$$

We have thus obtained

$$
\begin{aligned}
\left(1+\frac{1}{N}\right) & \left(1+O\left(\frac{1}{N^{2}}\right)\right) \\
= & \frac{\left(1-x_{N, k}^{2}\right)^{N+1}}{2 \sqrt{N \pi} x_{N, k}}\left(1-\frac{1-x_{N, k}^{2}}{2(N+2) x_{N, k}^{2}}+O\left(\frac{1}{N^{2} x_{N, k}^{4}}\right)\right) \\
= & 1-\frac{\lambda_{N, k}}{N}-\frac{1}{2 N} \lambda_{N, k}^{2}-\frac{i e^{-i \varphi_{N, k}}}{4 N \sin \varphi_{N, k}} \lambda_{N, k}+N v e^{2 i \varphi_{N, k}} \\
& +\frac{i e^{-i \varphi_{N, k}}}{4 N \sin \varphi_{N, k}}+O\left(\frac{(\log k)^{3}}{k^{2}}\right)
\end{aligned}
$$

which gives the value of $v$ with an $O\left((\log k)^{3} /\left(N k^{2}\right)\right)$ error.

As a corollary, we find a further development of $\gamma_{k}$, which is exactly the formula given in [3]: 
Corollary. If $\mu_{k}=(2 k-1 / 4) \pi$, then

$$
\begin{gathered}
\gamma_{k}=e^{-3 i \pi / 4} \sqrt{\mu_{k}}\left(1-\frac{i}{2 \mu_{k}} \log 2 \sqrt{\pi \mu_{k}}-\frac{1}{4 \mu_{k}^{2}} \log 2 \sqrt{\pi \mu_{k}}+\frac{1}{4 \mu_{k}^{2}}\right. \\
\left.+\frac{1}{8 \mu_{k}^{2}}\left(\log 2 \sqrt{\pi \mu_{k}}\right)^{2}+O\left(\frac{(\log k)^{3}}{k^{3}}\right)\right) .
\end{gathered}
$$

Proof. From (31) and (49), we get

$$
\begin{aligned}
1-\frac{\bar{\gamma}_{k}^{2}}{N}= & \left(1-i \frac{\mu_{k}}{N}\right)\left(1+\frac{1}{N} \log 2 \sqrt{\pi} \sqrt{\mu_{k}}+\frac{i}{2 N \mu_{k}}\left(\log 2 \sqrt{\pi} \sqrt{\mu_{k}}-1\right)\right) \\
& +O\left(\frac{(\log k)^{3}}{N k^{2}}\right)
\end{aligned}
$$

hence

$$
\gamma_{k}^{2}=-i \mu_{k}-\log 2 \sqrt{\pi \mu_{k}}+\frac{i}{2 \mu_{k}} \log 2 \sqrt{\pi \mu_{k}}-\frac{i}{2 \mu_{k}}+O\left(\frac{(\log k)^{3}}{k^{2}}\right)
$$

and

$$
\begin{aligned}
\gamma_{k}=\sqrt{-i \mu_{k}}( & 1-\frac{i}{2 \mu_{k}} \log 2 \sqrt{\pi \mu_{k}}-\frac{1}{4 \mu_{k}^{2}} \log 2 \sqrt{\pi \mu_{k}} \\
& \left.+\frac{1}{4 \mu_{k}^{2}}+\frac{1}{8 \mu_{k}^{2}}\left(\log 2 \sqrt{\pi \mu_{k}}\right)^{2}+O\left(\frac{(\log k)^{3}}{k^{3}}\right)\right)
\end{aligned}
$$

and the corollary is proved.

\section{Small roots of $Q_{N}$ : further estimates.}

We are now able to give a much better estimate for the small roots of $Q_{N}$. Indeed, we used the rough estimate $\left|e^{-N x_{N, k}^{2}}\right| \leq e^{N\left|x_{N, k}^{2}\right|}$ which is far from being good since $x_{N, k}$ accumulates on the line $x=y$ for $k$ big (and $k^{2}=O(N)$ ), so that $e^{-N x_{N, k}^{2}}$ is much smaller than $e^{N\left|x_{N, k}\right|^{2}}$ : indeed if $k^{2}=O(N)$ we find that

$$
x_{N, k}^{2}=-\frac{1}{N} \log 2 \sqrt{\pi\left(2 k-\frac{1}{4}\right) \pi}+\frac{i}{N}\left(2 k-\frac{1}{4}\right) \pi+O\left(\frac{\log k}{N k}\right),
$$


hence

$$
\begin{aligned}
\left|e^{-N x_{N, k}^{2}}\right| & =e^{\log 2 \sqrt{\pi(2 k-1 / 4) \pi}} e^{O(\log k / k)} \\
& =2 \sqrt{\pi} \sqrt{\left(2 k-\frac{1}{4}\right) \pi}\left(1+O\left(\frac{\log k}{k}\right)\right),
\end{aligned}
$$

while

$$
e^{N\left|x_{N, k}\right|^{2}} \geq e^{(2 k-1 / 4) \pi}\left(1+O\left(\frac{\log k}{k}\right)\right) .
$$

Thus, we may improve Result 4 in an impressive manner: for a much bigger set of indexes $k,-\bar{\gamma}_{k} / \sqrt{N}$ provides a very precise approximation of $x_{N, k}$ :

Result 8. There exist $\eta_{0}>0$ and $C_{0}>0$ so that for $N$ large enough and $k \leq \eta_{0} N^{1 / 5} /(\log N)^{2 / 5}$ we have

$$
\left|x_{N, k}+\frac{\bar{\gamma}_{k}}{\sqrt{N}}\right| \leq C_{0} \frac{1}{N \sqrt{N}}\left(\frac{k^{5 / 2}}{1+\log k}\right)
$$

ProOF. We write

$\tilde{Q}_{N}(t)=4 \sqrt{\frac{N}{\pi}} \frac{4^{N}(N !)^{2}}{(2 N+1) !} Q_{N}(t)=1+O\left(\frac{1}{N^{2}}\right)+2 \sqrt{\frac{N}{\pi}} \int_{0}^{t}\left(1-s^{2}\right)^{N} d s$

and approximate $\left(1-s^{2}\right)^{N}$ by $e^{-N s^{2}}$ (provided that $N t^{4}$ remains bounded: $\left.\left|N t^{4}\right| \leq A_{0}\right)$

$$
\left(1-s^{2}\right)^{4}=e^{N \log \left(1-s^{2}\right)}=e^{-N s^{2}}\left(1+O\left(N s^{4}\right)\right) .
$$

Thus

$$
\tilde{Q}_{N}(t)=\operatorname{erfc}(-\sqrt{N} t)+O\left(\frac{1}{N^{2}}\right)+\sqrt{N} \int_{0}^{t} e^{-N s^{2}} O\left(N s^{4}\right) d s .
$$

Let $\theta=\operatorname{Arg} t$ and assume $\theta \in(\pi / 4, \pi / 2)$. Then we have

$$
\begin{aligned}
\left|\sqrt{N} \int_{0}^{t} e^{-N s^{2}} O\left(N s^{4}\right) d s\right| & \leq C N \sqrt{N}|t|^{3} \int_{0}^{|t|} e^{-N \lambda^{2} \cos 2 \theta} \lambda d \lambda \\
& \leq C \frac{\left|e^{-N t^{2}}\right| \sqrt{N}|t|^{3}}{2|\cos 2 \theta|} .
\end{aligned}
$$


We have thus proved that for $\left|N t^{4}\right| \leq A_{0}$ and $\operatorname{Arg} t \in(\pi / 4, \pi / 2)$ we have

$$
\left|\tilde{Q}_{N}(t)-\operatorname{erfc}(-\sqrt{N} t)\right| \leq C\left(\frac{1}{N^{2}}+\sqrt{N}|t|^{3} \frac{\left|e^{-N t^{2}}\right|}{2|\cos 2 \operatorname{Arg} t|}\right)
$$

Now, we write $t=x_{N, k}+\delta,|\delta| \leq \delta_{0} / N$. Remember that we have

$$
\left|x_{N, k}\right| \approx \sqrt{\frac{\left(2 k-\frac{1}{4}\right) \pi}{N}}
$$

(hence we will look at $k \leq \sqrt{A_{0} N /(2 \pi)}$ ) and

$$
\begin{aligned}
\operatorname{Arg} x_{N, k}= & \frac{\pi}{4}-\frac{1}{2} \varphi_{N, k}+\operatorname{Arg}\left(1+\frac{i e^{-i \varphi_{N, k}}}{4 N \sin \varphi_{N, k}} \log \left(2 \sqrt{2 N \pi \sin \varphi_{N, k}}\right)\right) \\
& +O\left(\frac{(\log k)}{k^{2}}\right) \\
= & \frac{\pi}{4}+\frac{\log \left(2 \sqrt{\pi} \sqrt{\left(2 k-\frac{1}{4}\right) \pi}\right)}{2\left(2 k-\frac{1}{4}\right) \pi}+O\left(\frac{(\log k)^{2}}{k^{2}}\right)+O\left(\frac{k}{N}\right),
\end{aligned}
$$

hence if $k \geq k_{0}$ where $k_{0}$ is large enough so that

$$
O\left(\frac{(\log k)^{2}}{k^{2}}\right)+O\left(\frac{k}{N}\right)=O\left(\frac{(\log k)^{2}}{k^{2}}\right)+O\left(\frac{1}{k}\right)
$$

is smaller than

$$
\frac{1}{2} \frac{\log 2 \sqrt{\pi} \sqrt{\left(2 k-\frac{1}{4}\right) \pi}}{2\left(2 k-\frac{1}{4}\right) \pi}
$$

we find that $\operatorname{Arg} x_{N, k} \in(\pi / 4, \pi / 2)$. (This is also true for $k \leq k_{0}$, if $N$ is large enough, since $\left.x_{N, k} \sim-\bar{\gamma}_{k} / \sqrt{N}\right)$. 
Moreover

$\cos \left(2 \operatorname{Arg} x_{N, k}\right)$

$$
\begin{aligned}
& =-\sin \left(\frac{\log \left(2 \sqrt{\pi} \sqrt{\left(2 k-\frac{1}{4}\right) \pi}\right)}{\left(2 k-\frac{1}{4}\right) \pi}+O\left(\frac{(\log k)^{2}}{k^{2}}\right)+O\left(\frac{k}{N}\right)\right) \\
& =-\frac{\log \left(2 \sqrt{\pi} \sqrt{\left(2 k-\frac{1}{4}\right) \pi}\right)}{\left(2 k-\frac{1}{4}\right) \pi}+O\left(\frac{(\log k)^{2}}{k^{2}}\right)+O\left(\frac{k}{N}\right),
\end{aligned}
$$

hence $\cos \left(2 \operatorname{Arg} x_{N, k}\right)$ has order of magnitude $\log k / k$. Thus we obtain for $\delta_{0}$ small enough

$$
\begin{aligned}
& \text { - } t=x_{N, k}\left(1+O\left(\frac{1}{\sqrt{N k}}\right)\right), \\
& \text { - } e^{-N t^{2}}=e^{-N x_{N, k}^{2}}\left(1+O\left(\sqrt{\frac{k}{N}}\right)+O\left(\frac{1}{N}\right)\right), \\
& \text { - } \operatorname{Arg} t=\operatorname{Arg} x_{N, k}+O\left(\frac{1}{\sqrt{N k}}\right)=\operatorname{Arg} x_{N, k}+O\left(\frac{1}{k \sqrt{k}}\right),
\end{aligned}
$$

thus we have

$$
\begin{aligned}
\left|\tilde{Q}_{N}(t)-\operatorname{erfc}(-\sqrt{N} t)\right| & \leq C\left(\frac{1}{N^{2}}+\sqrt{N}\left(\frac{k}{N}\right)^{3 / 2} \frac{\sqrt{k}}{(\log k) / k}\right) \\
& \leq C^{\prime} \frac{k^{3}}{N \log k} .
\end{aligned}
$$

On the other hand we have

$$
\begin{aligned}
\mid \operatorname{erfc}(-\sqrt{N} t) & -\operatorname{erfc}\left(-\sqrt{N} x_{N, k}\right) \mid \\
& =\left|2 \sqrt{\frac{N}{\pi}} \int_{x_{N, k}}^{t} e^{-N s^{2}} d s\right| \\
& =\left|e^{-N x_{N, k}^{2}}\right| 2 \sqrt{\frac{N}{\pi}}\left|\int_{0}^{\delta} e^{-2 N x_{N, k} s-N s^{2}} d s\right| .
\end{aligned}
$$

We notice that

$$
\left|2 N x_{N, k} s+N s^{2}\right| \leq 2\left|x_{N, k}\right| \delta_{0}+\frac{\delta_{0}^{2}}{N} \leq C \frac{\delta_{0}}{\sqrt{N}},
$$


so that if $N$ is large enough,

$$
\left|e^{-2 N x_{N, k} s-N s^{2}}-1\right| \leq \frac{1}{2},
$$

which gives

$\left|\operatorname{erfc}(-\sqrt{N} t)-\operatorname{erfc}\left(-\sqrt{N} x_{N, k}\right)\right| \geq 2 \sqrt{\frac{N}{\pi}}\left|e^{-N x_{N, k}^{2}}\right| \frac{1}{2}|\delta| \geq C \sqrt{N k}|\delta|$

Thus

$$
\left\{\begin{array}{l}
|\operatorname{erfc}(-\sqrt{N} t)| \geq C_{1} \sqrt{N} k \delta-C_{2} \frac{k^{3}}{N \log k} \\
\left|\operatorname{erfc}(-\sqrt{N} t)-\tilde{Q}_{N}(t)\right| \leq C_{2} \frac{k^{3}}{\sqrt{N} \log k}
\end{array}\right.
$$

Now choose

$$
\delta_{N, k}=\frac{3 C_{2}}{C_{1}} \frac{k^{5 / 2}}{N^{3 / 2} \log k}
$$

(we have $\delta_{N, k}<\delta_{0} / N$ if $k^{5 / 2} / \log k<\delta_{0} C_{1} \sqrt{N} /\left(3 C_{2}\right)$ ); we obtain that

$$
\sup _{\left|t-x_{N, k}\right|=\delta_{N, k}}\left|\operatorname{erfc}(-\sqrt{N} t)-\tilde{Q}_{N}(t)\right| \leq \frac{1}{2} \inf _{\left|t-x_{N, k}\right|=\delta_{N, k}}|\operatorname{erfc}(-\sqrt{N} t)|,
$$

hence by Rouché's theorem we find that $\tilde{Q}_{N}$ and $\operatorname{erfc}(-\sqrt{N} t)$ have the same number of roots in the disk $\left|t-x_{N, k}\right|<\delta_{N, k}$. Since

$$
\left|x_{N, k}-x_{N, k+1}\right| \approx \sqrt{\frac{\pi}{2 k N}}
$$

and

$$
\sqrt{k N} \delta_{N, k}=O\left(\frac{k^{3}}{N \log k}\right)=O\left(\frac{1}{N^{2 / 5}(\log N)^{7 / 5}}\right)=o(1)
$$

(if $\left.k \leq C N^{1 / 5} /(\log N)^{2 / 5}\right)$, we find: for $k \leq \eta_{0} N^{1 / 5} /(\log N)^{2 / 5}\left(\eta_{0}\right.$ small enough)

$$
\left|x_{N, k}+\frac{\bar{\gamma}_{k}}{\sqrt{N}}\right| \leq C \frac{1}{N \sqrt{N}}\left(\frac{k^{5 / 2}}{\log k}\right)
$$


Result 8 is proved.

Result 8 is enough for what we want to prove. But, of course, we may develop a bit further $\left(1-s^{2}\right)^{N}$ and get a better approximation for $x_{N, k}$ :

Result 9. For $k \leq \eta_{0} N^{1 / 5} /(\log N)^{2 / 5}$ we have more precisely

$$
x_{N, k}=-\frac{\bar{\gamma}_{k}}{\sqrt{N}}+\frac{1}{N \sqrt{N}}\left(\frac{1}{2} \bar{\gamma}_{k}^{3}+\frac{3}{8} \bar{\gamma}_{k}+O(\sqrt{\log k})\right) \text {. }
$$

Proof. We write $\log \left(1-s^{2}\right)=-s^{2}-s^{4} / 2+O\left(s^{6}\right)$. Hence we have

$$
\left(1-s^{2}\right)^{N}=e^{-N s^{2}}\left(1-N \frac{s^{4}}{2}+O\left(N s^{6}\right)+O\left(N^{2} s^{8}\right)\right),
$$

provided that $|s| \leq A_{0} / N^{1 / 4}$.

Thus we have for $|t| \leq A_{0} / N^{1 / 4}$ and $\operatorname{Arg} t \in(\pi / 4, \pi / 2)$

$$
\begin{aligned}
& \mid \tilde{Q}_{N}(t)-\left.\operatorname{erfc}(-\sqrt{N} t)+2 \sqrt{\frac{N}{\pi}} N \int_{0}^{t} e^{-N s^{2}} s^{4} d s\right) \\
& \leq C\left(\frac{1}{N^{2}}+\sqrt{N}\left|\frac{t^{5} e^{-N t^{2}}}{\cos (2 \operatorname{Arg} t)}\right|+N \sqrt{N}\left|\frac{t^{7} e^{-N t^{2}}}{\cos (2 \operatorname{Arg} t)}\right|\right) .
\end{aligned}
$$

Moreover we have

$$
\begin{aligned}
N \int_{0}^{t} e^{-N s^{2}} s^{4} d s & =\left[\frac{-e^{-N s^{2}} s^{3}}{2}\right]_{0}^{t}+\frac{3}{2} \int_{0}^{t} e^{-N s^{2}} s^{2} d s \\
& =\frac{-e^{-N t^{2}} t^{3}}{2}-\frac{3}{4 N} e^{-N t^{2}} t+\frac{3}{4 N} \int_{0}^{t} e^{-N s^{2}} d s .
\end{aligned}
$$

Now, we write $\eta=1 / \sqrt{2 N|\cos (2 \operatorname{Arg} t)|}$ (if $t \approx x_{N, k}$, we have $\eta \approx$ $\sqrt{4 k /(N \log k)}<|t|)$ and we write

$$
\begin{aligned}
\left|\int_{0}^{t} e^{-N s^{2}} d s\right| & \leq \int_{0}^{\eta}\left|e^{-N t^{2}}\right| d s+\int_{\eta}^{|t|} e^{-N s^{2} \cos (2 \operatorname{Arg} t)} \frac{s d s}{\eta} \\
& \leq \eta\left|e^{-N t^{2}}\right|+\frac{\left|e^{-N t^{2}}\right|}{2 N|\cos (2 \operatorname{Arg} t)| \eta} \\
& =\frac{2\left|e^{-N t^{2}}\right|}{\sqrt{2 N|\cos (2 \operatorname{Arg} t)|}}
\end{aligned}
$$


Finally we get

$$
\begin{aligned}
\operatorname{erfc}\left(-\sqrt{N} x_{N, k}\right)= & e^{-N x_{N, k}^{2}} \sqrt{\frac{N}{\pi}} x_{N, k}^{3}+e^{-N x_{N, k}^{2}} \frac{3}{4 \sqrt{N \pi}} x_{N, k} \\
& +O\left(\frac{1}{N^{2}}\right)+O\left(\frac{k^{4}}{N^{2} \log k}\right)+O\left(\frac{k^{5}}{N^{2} \log k}\right) \\
& +O\left(\frac{\sqrt{\log k}}{N}\right)
\end{aligned}
$$

and, assuming again $k<\eta_{0} N^{1 / 5} /(\log N)^{2 / 5}$,

$$
\operatorname{erfc}\left(-\sqrt{N} x_{N, k}\right)=e^{-N x_{N, k}^{2}} \sqrt{\frac{N}{\pi}} x_{N, k}^{3}\left(1+O\left(\frac{1}{k}\right)\right)
$$

On the other hand, we have $x_{N, k}=-\bar{\gamma}_{k} / \sqrt{N}+s$ with

$$
s=O\left(\frac{1}{N \sqrt{N}} \frac{k^{5 / 2}}{\log k}\right)
$$

and we want a better estimate for $s$. We have

$$
\sqrt{N} s \bar{\gamma}_{k}=O\left(\frac{1}{N} \frac{k^{3}}{\log k}\right)=O\left(\frac{1}{N^{2 / 5}}\right)
$$

and thus we may develop

$$
\begin{aligned}
\operatorname{erfc}\left(\bar{\gamma}_{k}-\sqrt{N} s\right) & =e^{-\bar{\gamma}_{k}^{2}} \frac{2}{\sqrt{\pi}} \int_{0}^{-\sqrt{N} s} e^{-2 \bar{\gamma}_{k} u-u^{2}} d u \\
& =-\frac{2}{\sqrt{\pi}} e^{-\bar{\gamma}_{k}^{2}} \sqrt{N} s\left(1+O\left(\sqrt{N} s \bar{\gamma}_{k}\right)+O\left(N s^{2}\right)\right)
\end{aligned}
$$

Hence we find

$$
-\frac{2}{\sqrt{\pi}} e^{-\bar{\gamma}_{k}^{2}} \sqrt{N} s \sim \sqrt{\frac{N}{\pi}} x_{N, k}^{3} e^{-N x_{N, k}^{2}}
$$

and therefore

$$
s \sim-\frac{1}{2} x_{N, k}^{3}=O\left(\frac{k^{3 / 2}}{N^{3 / 2}}\right),
$$


so that

$$
\begin{aligned}
-e^{-\bar{\gamma}_{k}^{2}} & \frac{2}{\sqrt{\pi}} \sqrt{N} s\left(1+O\left(\frac{k^{2}}{N}\right)+O\left(\frac{k^{3}}{N^{2}}\right)\right) \\
& =\sqrt{\frac{N}{\pi}} x_{N, k}^{3} e^{-N x_{N, k}^{2}}+\frac{3}{4 \sqrt{N \pi}} x_{N, k} e^{-N x_{N, k}^{2}}+O\left(\frac{\sqrt{\log k}}{N}\right)
\end{aligned}
$$

so that $\left(\right.$ since $\left.e^{-N x_{N, k}^{2}+\bar{\gamma}_{k}^{2}}=1+O\left(\sqrt{N} s \bar{\gamma}_{k}\right)=1+O\left(k^{2} / N\right)\right)$

$$
\begin{aligned}
s & =-\frac{1}{2} x_{N, k}^{3}-\frac{3}{8 N} x_{N, k}+O\left(\frac{\sqrt{\log k}}{N \sqrt{N}}\right) \\
& =\frac{1}{2} \frac{\bar{\gamma}_{k}^{3}}{N \sqrt{N}}+\frac{3 \bar{\gamma}_{k}}{8 N \sqrt{N}}+O\left(\frac{\sqrt{\log k}}{N \sqrt{N}}\right)
\end{aligned}
$$

and Result 9 is proved.

\section{The phase of a general Daubechies filter.}

We have now almost achieved the proof of Theorem 1. Indeed, we have given estimates for $x_{N, k}$, hence for $z_{N, k}$, which is the solution of $x_{N, k}=\left(z_{N, k}+1 / z_{N, k}\right) / 2$ with $\operatorname{Re} z_{N, k}>0$, hence which is given by $z_{N, k}=x_{N, k}+\sqrt{x_{N, k}^{2}-1}$. We thus have proved:

Proposition 3. Let $P_{N}$ be the $N$-th polynomial of I. Daubechies

$$
P_{N}(z)=\left(\frac{1+z}{2}\right)^{2 N+2} \sum_{k=0}^{N}(-1)^{k}\left(\begin{array}{c}
N+k \\
k
\end{array}\right)\left(\frac{1-z}{2}\right)^{2 k}
$$

which is related to $Q_{N}$ by

$$
e^{i(2 N+1) \xi} P_{N}\left(e^{-i \xi}\right)=Q_{N}(\cos \xi)
$$

or equivalently

$$
P_{N}(z)=z^{2 N+1} Q_{N}\left(\frac{1}{2}\left(z+\frac{1}{z}\right)\right) .
$$

Then the roots of $P_{N}$ are precisely given as the following ones:

- $z=-1$ with multiplicity $2 N+2$, 
- $2 N$ roots with multiplicity 1 which can be decomposed into

$$
\left\{z_{N, k}, \overline{z_{N, k}}, \frac{1}{z_{N, k}}, \frac{1}{\bar{z}_{N, k}}\right\}_{1 \leq k \leq[N / 2]},
$$

(together with $\left\{z_{N,(N+1) / 2}, 1 / z_{N,(N+1) / 2}\right\}$ if $N$ is odd), where $\operatorname{Im} z_{N, k} \geq$ $0, \operatorname{Re} z_{N, k} \geq 0,\left|z_{N, k}\right|>1, \operatorname{Im} z_{N, k}>0$ for $k<[(N+1) / 2]$ and $\operatorname{Im} z_{N,(N+1) / 2}=0$.

Moreover we have, for $N$ large enough:

- if $k \leq \eta_{0} N^{1 / 5} /(\log N)^{2 / 5}$ (where $\eta_{0}$ is fixed independently of $N$ and is small enough)

$$
z_{N, k}=i-\frac{\bar{\gamma}_{k}}{\sqrt{N}}+O\left(\frac{k}{N}\right),
$$

where $\gamma_{k}$ is the $k$-th zero $\gamma$ of $\operatorname{erfc}(z)$ with $\operatorname{Im} \gamma>0$

- for all $k$

$$
z_{N, k}=y_{N, k}+\sqrt{y_{N, k}^{2}-1}+O\left(\frac{1+\log k}{k \sqrt{N k}}\right)
$$

where

$$
\begin{aligned}
y_{N, k}= & \left(1-e^{-2 i(8 k-1) \pi /(8 N+6)}\right. \\
& \left.\quad-\frac{1}{N} e^{-2 i(8 k-1) \pi /(8 N+6)} \log 2 \sqrt{2 N \pi \sin \left(\frac{8 k-1}{8 N+6} \pi\right)}\right)^{1 / 2} .
\end{aligned}
$$

ProOF. Just write $z_{N, k}=x_{N, k}+\sqrt{x_{N, k}^{2}-1}$ and apply results 6 and 8.

Of course, we could give better estimates using results 7 and 9 , but we won't need them. We have easy estimates for $1 / z_{N, k}$ as well since $1 / z_{N, k}=x_{N, k}-\sqrt{x_{N, k}^{2}-1}$.

We are now going to use proposition 3 in the estimation of the phase of a Daubechies filter. We want to approximate for $\xi \in[-\pi, \pi]$, $1 /\left(e^{-i \xi}-\lambda_{N, k}\right)$ where

$$
\lambda_{N, k} \in\left\{z_{N, k}, \frac{1}{z_{N, k}}, \bar{z}_{N, k}, \frac{1}{\bar{z}_{N, k}}\right\} .
$$


A direct consequence of Proposition 3 is the following proposition:

Proposition 4. Let $\xi \in[-\pi, \pi]$ and let $z_{N, k}, 1 \leq k \leq[(N+1) / 2]$ be the roots of $P_{N}$ described in Proposition 3. Let $\lambda_{N, k} \in\left\{z_{N, k}, 1 / z_{N, k}, \bar{z}_{N, k}\right.$, $\left.1 / \bar{z}_{N, k}\right\}$. Then

i) for $1 \leq k \leq \eta_{0} N^{1 / 5} /(\log N)^{2 / 5}$ we have, writing $\widetilde{z_{N, k}}=i-$ $\bar{\gamma}_{k} / \sqrt{N}$

$$
\left|\frac{1}{e^{-i \xi}-\lambda_{N, k}}-\frac{1}{e^{-i \xi}-\widetilde{\lambda_{N, k}}}\right| \leq C \frac{k}{N} \frac{1}{\frac{k}{N}+|\cos \xi|^{2}},
$$

where $C$ doesn't depend neither on $N$ nor on $k$ nor on $\xi$ (and where $\widetilde{\lambda_{N, k}}=\widetilde{z_{N, k}}$ if $\lambda_{N, k}=z_{N, k}, 1 / \widetilde{z_{N, k}}$, if $\lambda_{N, k}=1 / z_{N, k}$ and so on ...).

ii) for $k \geq k_{0}\left(k_{0}\right.$ large enough independently of $\left.N\right)$ we have, writing $\widehat{z_{N, k}}=y_{N, k}+\sqrt{y_{N, k}^{2}-1}$ as in formula $(58)$,

$$
\left|\frac{1}{e^{-i \xi}-\lambda_{N, k}}-\frac{1}{e^{-i \xi}-\widehat{\lambda_{N, k}}}\right| \leq C \frac{\log k}{k \sqrt{N k}} \frac{1}{\frac{k}{N}+|\cos \xi|^{2}} .
$$

Proof. Of course, we may assume $\xi \in[0, \pi]$. If $\xi \in[\pi / 2, \pi]$, the estimation is easy since $\operatorname{Re} e^{-i \xi}<0$ and $\operatorname{Re} \lambda_{N, k}>0$ (as well $\operatorname{Re} \widehat{\lambda_{N, k}}$ and $\left.\operatorname{Re} \widetilde{\lambda_{N, k}}\right)$. Thus,

$$
\left|e^{-i \xi}-\lambda_{N, k}\right| \geq \operatorname{Re}\left(-e^{-i \xi}+\lambda_{N, k}\right) \geq C \sqrt{\frac{k}{N}}+|\cos \xi|
$$

and the same for $\left|e^{-i \xi}-\widehat{\lambda_{N, k}}\right|$ and $\left|e^{-i \xi}-\widetilde{\lambda_{N, k}}\right|$. Of course, we must prove that $\min \left\{\operatorname{Re} \lambda_{N, k}, \operatorname{Re} \widehat{\lambda_{N, k}}, \operatorname{Re} \widetilde{\lambda_{N, k}}\right\} \geq C \sqrt{k / N}$. For $\operatorname{Re} \widetilde{\lambda_{N, k}}$, it is obvious, since

$$
\operatorname{Re} \widetilde{\lambda_{N, k}} \geq \frac{-\operatorname{Re} \gamma_{k}}{\sqrt{N}\left|i-\frac{\bar{\gamma}_{k}}{\sqrt{N}}\right|^{2}} \approx \sqrt{\frac{k \pi}{N}}
$$

For $\operatorname{Re} \lambda_{N, k}$, if $k<\eta_{0} N^{1 / 5} /(\log N)^{2 / 5}$, we deduce that $\operatorname{Re} \lambda_{N, k} \geq$ $C \sqrt{k / N}$ since

$$
\left|\lambda_{N, k}-\widetilde{\lambda_{N, k}}\right| \leq\left|z_{N, k}-\widetilde{z_{N, k}}\right| \leq C \frac{k}{N} \leq \sqrt{\frac{k}{N}} C^{\prime} N^{-2 / 5} .
$$


We thus turn our attention to $\operatorname{Re} \widehat{\lambda_{N, k}} \geq \operatorname{Re} \widehat{z_{N, k}} /\left|\widehat{z_{N, k}}\right|^{2}$ and $\operatorname{Re} \lambda_{N, k} \geq$ $\operatorname{Re} z_{N, k} /\left|z_{N, k}\right|^{2}$ for large $k$ 's. We define $\mu_{N, k}=\sqrt{1-e^{-2 i(8 k-1) \pi /(8 N+6)}}$ and $\xi_{N, k}=\mu_{N, k}+\sqrt{\mu_{N, k}^{2}-1}$. We have

$$
\begin{aligned}
\xi_{N, k}= & \sqrt{2 \sin \left(\frac{8 k-1}{8 N+6}\right)} e^{i(\pi / 4-(8 k-1) \pi /(2(8 N+6)))} \\
& +e^{i(\pi / 2-(8 k-1) \pi /(8 N+6))} \\
= & 1+\sqrt{2} e^{i(\pi / 4-(8 k-1) \pi /(2(8 N+6))+\arcsin \sqrt{2} \sin (\pi / 4-(8 k-1) \pi / 2(8 N+6)))}
\end{aligned}
$$

and thus we study $1+\sqrt{2} e^{i(\omega+\arcsin \sqrt{2} \sin \omega)}$ for $\omega \in[0, \pi / 4]$. We have

$$
\begin{aligned}
\operatorname{Re}(1+\sqrt{2} & \left.e^{i(\omega+\arcsin \sqrt{2} \sin \omega)}\right) \\
& =\sqrt{1-2 \sin ^{2} \omega}\left(\sqrt{1-2 \sin ^{2} \omega}+\sqrt{2 \cos ^{2} \omega}\right) \\
& =\sqrt{\cos 2 \omega}\left(\sqrt{2 \cos ^{2} \omega}+\sqrt{1-2 \sin ^{2} \omega}\right) \geq \sqrt{\frac{4}{\pi}\left(\frac{\pi}{4}-\omega\right)},
\end{aligned}
$$

which gives

$$
\operatorname{Re} \xi_{N, k} \geq \sqrt{2 \frac{8 k-1}{8 N+6}} \geq \sqrt{\frac{k}{N}}
$$

Now we have

$$
\left|\widehat{z_{N, k}}-\xi_{N, k}\right| \leq C \sqrt{\frac{k}{N}} \frac{\log k}{k}
$$

so that if $k$ is large enough we have

$$
\operatorname{Re} \widehat{z_{N, k}} \geq C^{\prime} \sqrt{\frac{k}{N}} .
$$

Moreover

$$
\left|z_{N, k}-\widehat{z_{N, k}}\right| \leq C \sqrt{\frac{k}{N}} \frac{\log k}{k^{2}}
$$

and thus

$$
\operatorname{Re} z_{N, k} \geq C^{\prime \prime} \sqrt{\frac{k}{N}}
$$

Finally, we control $\left|z_{N, k}\right|$ and $\left|\widehat{z_{N, k}}\right|$ by

$$
\left|z_{N, k}\right|+\left|\widehat{z_{N, k}}\right| \leq 1+\sqrt{2}+O\left(\sqrt{\frac{k}{N}} \frac{\log k}{k}\right) \leq C .
$$


Thus we obtain

$$
\operatorname{Re} \lambda_{N, k} \geq C \sqrt{\frac{k}{N}} \quad \text { and } \quad \operatorname{Re} \widehat{\lambda_{N, k}} \geq C \sqrt{\frac{k}{N}}
$$

We are going to prove that

$$
\left|e^{-i \xi}-\lambda_{N, k}\right| \geq C\left(\sqrt{\frac{k}{N}}+|\cos \xi|\right)
$$

and

$$
\left|e^{-i \xi}-\widehat{\lambda_{N, k}}\right| \geq C\left(\sqrt{\frac{k}{N}}+|\cos \xi|\right)
$$

holds for $\xi \in[0, \pi / 2]$ as well. Notice that if $\left|\lambda_{N, k}\right|<1$, we have

$$
\left|\lambda_{N, k}-e^{-i \xi}\right|=\left|\frac{1}{z_{N, k}}\right|\left|e^{-i \xi}-\frac{1}{\overline{\lambda_{N, k}}}\right| \geq \frac{1}{C^{\prime}}\left|e^{-i \xi}-\frac{1}{\overline{\lambda_{N, k}}}\right|
$$

(and the same for $\left|e^{-i \xi}-\widehat{\lambda_{N, k}}\right|$ ) so that we may assume $\left|\lambda_{N, k}\right|>1$. If $\lambda_{N, k}=z_{N, k}$, our equality is obvious: for $\xi_{N, k}$ we have either $\operatorname{Im} \xi_{N, k} \geq 1$ or $\operatorname{Re} \xi_{N, k} \geq 2$ and, since $\operatorname{Im} e^{-i \xi}<0$, we find $\left|e^{-i \xi}-\xi_{N, k}\right| \geq 1$, hence (for $k$ large), $\left|e^{-i \xi}-z_{N, k}\right| \geq 1 / 2$ and $\left|e^{-i \xi}-\widehat{z_{N, k}}\right| \geq 1 / 2$, while

$$
\frac{1}{2} \geq \frac{1}{4}\left(\sqrt{\frac{k}{N}}+|\cos \xi|\right) .
$$

Now if $\lambda_{N, k}$ is the conjugate of $z_{N, k}$ or $\widehat{z_{N, k}}$, we are going to show that

$$
\left|e^{-i \xi}-\bar{\xi}_{N, k}\right| \geq C\left(\sqrt{\frac{k}{N}}+|\cos \xi|\right)
$$

which gives the control over $\left|e^{-i \xi}-\lambda_{N, k}\right|$ for large $k$ 's. Thus we are led to show that

$$
\left\{\begin{aligned}
\text { for } \xi \in\left[0, \frac{\pi}{2}\right] \text { and } \omega \in\left[0, \frac{\pi}{4}\right] & \\
\mid e^{-i \xi}-1-\sqrt{2} & e^{-i(\omega+\arcsin \sqrt{2} \sin \omega) \mid} \\
& \geq C\left(|\cos \xi|+\sqrt{\frac{\pi}{4}-\omega}\right) .
\end{aligned}\right.
$$


We compute easily $\mu(\xi, \omega)=\left|e^{-i \xi}-1-\sqrt{2} e^{-i(\omega+\arcsin \sqrt{2} \sin \omega)}\right|^{2}$

$$
\begin{aligned}
\mu(\xi, \omega)= & \left(\cos \xi-\sqrt{1-2 \sin ^{2} \omega}\left(\sqrt{2} \cos \omega+\sqrt{1-2 \sin ^{2} \omega}\right)\right)^{2} \\
+ & \left(\sin \xi-\sqrt{2} \sin \omega\left(\sqrt{2} \cos \omega+\sqrt{1-2 \sin ^{2} \omega}\right)\right)^{2} \\
= & +\left(\sqrt{2} \cos \omega+\sqrt{1-2 \sin ^{2} \omega}\right)^{2} \\
& -2\left(\sqrt{2} \cos \omega+\sqrt{1-2 \sin ^{2} \omega}\right) \\
& \cdot\left(\cos \xi \sqrt{1-2 \sin ^{2} \omega}+\sin \xi \sqrt{2} \sin \omega\right) \\
= & \left(\sqrt{2} \cos \omega-1+\sqrt{1-2 \sin ^{2} \omega}\right)^{2} \\
& +2\left(\sqrt{2} \cos \omega+\sqrt{1-2 \sin ^{2} \omega}\right) \\
& \cdot(1-\cos (\xi-\arcsin (\sqrt{2} \sin \omega))) \\
\geq & 1-2 \sin ^{2} \omega+2(1-\cos (\xi-\arcsin (\sqrt{2} \sin \omega))) .
\end{aligned}
$$

We have

$$
1-2 \sin ^{2} \omega=\cos 2 \omega \geq \frac{2}{\pi}\left(\frac{\pi}{2}-2 \omega\right) .
$$

On the other hand, we have

$$
\begin{aligned}
1-\cos (\xi-\arcsin \sqrt{2} \sin \omega) & =2 \sin ^{2}\left(\frac{\xi}{2}-\frac{1}{2} \arcsin \sqrt{2} \sin \omega\right) \\
& \geq \frac{2}{\pi^{2}}|\xi-\arcsin \sqrt{2} \sin \omega|^{2}
\end{aligned}
$$

Moreover we have

$$
\frac{\pi}{2}-\arcsin \sqrt{2} \sin \omega=\arcsin \sqrt{\cos 2 \omega} \leq \frac{\pi}{2} \sqrt{\cos 2 \omega},
$$

hence we have (using $|a+b|^{2} \geq a^{2} / 3-b^{2} / 2$ )

$$
\begin{aligned}
\mu(\xi, \omega)^{2} & \geq \cos 2 \omega+\frac{4}{\pi^{2}}\left|\xi-\frac{\pi}{2}+\frac{\pi}{2}-\arcsin \sqrt{2} \sin \omega\right|^{2} \\
& \geq \cos 2 \omega+\frac{4}{3 \pi^{2}}\left|\xi-\frac{\pi}{2}\right|^{2}-\frac{2}{\pi^{2}}\left|\frac{\pi}{2}-\arcsin \sqrt{2} \sin \omega\right|^{2} \\
& \geq \frac{1}{2} \cos 2 \omega+\frac{4}{3 \pi^{2}} \cos ^{2} \xi \\
& \geq \frac{4}{3 \pi^{2}}\left(\cos ^{2} \xi+\left|\frac{\pi}{4}-\omega\right|\right)
\end{aligned}
$$


and thus (61) is proved.

Proposition 4 is then obvious since

$$
\left|\frac{1}{e^{-i \xi}-\lambda_{N, k}}-\frac{1}{e^{-i \xi}-\tilde{\lambda}_{N, k}}\right|=\frac{\left|\lambda_{N, k}-\tilde{\lambda}_{N, k}\right|}{\left|e^{-i \xi}-\lambda_{N, k}\right|\left|e^{-i \xi}-\tilde{\lambda}_{N, k}\right|}
$$

and since we control each term due to (61) or to Proposition 3.

We may now obtain Theorem 1 as a corollary of Proposition 4:

Corollary. With the same notation as in Proposition 4, if $k_{0} \leq k_{N} \leq$ $\eta_{0} N^{1 / 5} /(\log N)^{2 / 5}$ then

$$
\begin{array}{r}
\int_{0}^{2 \pi} \mid \sum_{k=1}^{[(N+1) / 2]} \frac{i e^{-i \xi}}{e^{-i \xi}-\lambda_{N, k}} \\
-\sum_{k=1}^{k_{N}} \frac{i e^{-i \xi}}{e^{-i \xi}-\tilde{\lambda}_{N, k}}-\sum_{k_{N}+1}^{[(N+1) / 2]} \frac{i e^{-i \xi}}{e^{-i \xi}-\hat{\lambda}_{N, k}} \mid d \xi \\
\leq C\left(\frac{k_{N}^{3 / 2}}{\sqrt{N}}+\frac{\log k_{N}}{k_{N}}\right) .
\end{array}
$$

Proof. Using Proposition 4, and writing $I_{N}(\xi)$ for

$$
I_{N}(\xi)=\sum_{k=1}^{N} \frac{i e^{-i \xi}}{e^{-i \xi}-\lambda_{N, k}}-\sum_{k=1}^{k_{N}} \frac{i e^{-i \xi}}{e^{-i \xi}-\tilde{\lambda}_{N, k}}-\sum_{k_{N}+1}^{[(N+1) / 2]} \frac{i e^{-i \xi}}{e^{-i \xi}-\tilde{\lambda}_{N, k}}
$$

we get

$$
I_{N}(\xi) \leq \sum_{k=1}^{k_{N}} C \frac{k}{N} \frac{1}{\frac{k}{N}+|\cos \xi|^{2}}+\sum_{k_{N}+1}^{[(N+1) / 2]} C \frac{\log k}{k \sqrt{N k}} \frac{1}{\frac{k}{N}+|\cos \xi|^{2}} .
$$

Thus we have to estimate

$$
\begin{aligned}
\int_{0}^{2 \pi} \frac{d \xi}{k+N|\cos \xi|^{2}} & \leq 4 \int_{0}^{\arccos \sqrt{k / N}} \frac{d \xi}{N \cos ^{2} \xi}+4 \int_{\arccos \sqrt{k / N}}^{\pi / 2} \frac{d \xi}{k} \\
& =\frac{4}{N} \tan \left(\arccos \sqrt{\frac{k}{N}}\right)+\frac{4}{k}\left(\frac{\pi}{2}-\arccos \sqrt{\frac{k}{N}}\right) \\
& \leq \frac{4}{\sqrt{N k}}+\frac{2 \pi}{\sqrt{N k}},
\end{aligned}
$$


so that

$$
\int_{0}^{2 \pi} I_{N}(\xi) d \xi \leq C^{\prime}\left(\sum_{k=1}^{k_{N}} \sqrt{\frac{k}{N}}+\sum_{k_{N}+1}^{[(N+1) / 2]} \frac{\log k}{k^{2}}\right) \leq C^{\prime \prime}\left(\frac{k_{N}^{3 / 2}}{\sqrt{N}}+\frac{\log k_{N}}{k_{N}}\right) .
$$

Now Theorem 1 is proved with $k_{N}=\left[N^{1 / 5} / \log N\right]$. At least, we have proved it for $\xi \in[0,2 \pi]$. But $\omega\left(z_{N, 1}^{\varepsilon_{1}}, \ldots, z_{N, N}^{\varepsilon_{N}}\right)-\omega\left(Z_{N, 1}^{\varepsilon_{1}}, \ldots, Z_{N, N}^{\varepsilon_{N}}\right)$ is $2 \pi$-periodical, since $\omega\left(Z_{1}, \ldots, Z_{N}\right)(\xi+2 \pi)-\omega\left(Z_{1}, \ldots, Z_{N}\right)(\xi)=2 i \pi M$ where $M$ is the number of $Z_{k}$ 's which lie inside the open disk $|Z|<1$.

\section{Minimum-phased Daubechies filters.}

This section is devoted to the proof of Theorem 2 .

Result 10. We have the following inequality

$$
\left|\frac{d}{d \xi} \omega\left(z_{N, 1}, \ldots, z_{N, N}\right)(\xi)-\frac{N}{2 \pi} \operatorname{Im} \int_{-\pi}^{\pi} \frac{i e^{-i \xi}}{e^{-i \xi}-\xi(\omega)} d \omega\right| \leq C \sqrt{N},
$$

where $\xi(\omega)=\sqrt{e^{-i \omega}}+\sqrt{1+e^{-i \omega}}$.

Proof. We approximate $z_{N, k}$ by $Z_{N, k}=Z((8 k-1) \pi /(8 N+6))$, $(1 \leq k \leq N)$ where

$$
Z(\omega)=\sqrt{2 \sin \omega} e^{i(\pi / 4-\omega / 2)}+e^{i(\pi / 2-\omega)} .
$$

We have shown that for $k_{0} \leq k \leq[(N+1) / 2]$, ( $k_{0}$ large enough) we have

$$
\left|\frac{1}{e^{-i \xi}-z_{N, k}}-\frac{1}{e^{-i \xi}-Z_{N, k}}\right| \leq C \frac{\log k}{\sqrt{N k}} \frac{1}{\frac{k}{N}+\cos ^{2} \xi}
$$

and

$$
\left|\frac{1}{e^{-i \xi}-\bar{z}_{N, k}}-\frac{1}{e^{-i \xi}-\bar{Z}_{N, k}}\right| \leq C \frac{\log k}{\sqrt{N k}} \frac{1}{\frac{k}{N}+\cos ^{2} \xi}
$$

(notice that $z_{N, N+1-k}=\bar{z}_{N, k}$ and $Z_{N, N+1-k}=\bar{Z}_{N, k}$ ). If $k<k_{0}$, we have to prove similarly

$$
\left|\frac{1}{e^{-i \xi}-z_{N, k}}-\frac{1}{e^{-i \xi}-Z_{N, k}}\right| \leq C \frac{1}{\sqrt{N}} \frac{1}{\frac{1}{N}+\cos ^{2} \xi}
$$


and

$$
\left|\frac{1}{e^{-i \xi}-\bar{z}_{N, k}}-\frac{1}{e^{-i \xi}-\bar{Z}_{N, k}}\right| \leq C \frac{1}{\sqrt{N}} \frac{1}{\frac{1}{N}+\cos ^{2} \xi} .
$$

We have of course

$$
\left|z_{N, k}-Z_{N, k}\right| \leq\left|z_{N, k}\right|+\left|Z_{N, k}\right| \leq \frac{C}{\sqrt{N}},
$$

so that we only have to check that

$$
\left|e^{-i \xi}-Z_{N, k}\right| \geq \frac{1}{C}\left(\frac{1}{\sqrt{N}}+|\cos \xi|\right)
$$

(which is an easy consequence of (61)) and that

$$
\left|e^{-i \xi}-z_{N, k}\right| \geq \frac{1}{C}\left(\frac{1}{\sqrt{N}}+|\cos \xi|\right) .
$$

If $|\xi+\pi / 2| \geq 3\left|\gamma_{k_{0}}\right| / \sqrt{N}$ and $\xi \in[-2 \pi, 0]$, we find

$$
e^{-i \xi}-z_{N, k}=2 e^{-i(\xi / 2+\pi / 4)} \sin \left(\frac{\xi}{2}+\frac{\pi}{4}\right)-\frac{\bar{\gamma}_{k}}{\sqrt{N}}+O\left(\frac{1}{N}\right),
$$

hence

$$
\begin{aligned}
\left|e^{-i \xi}-z_{N, k}\right| & \geq\left|\sin \left(\frac{\xi}{2}+\frac{\pi}{4}\right)\right|-\frac{\left|\gamma_{k_{0}}\right|}{\sqrt{N}}+O\left(\frac{1}{N}\right) \\
& \geq \frac{1}{2}\left|\sin \left(\frac{\xi}{2}+\frac{\pi}{4}\right)\right| \\
& \geq \max \left\{\frac{1}{4}|\cos \xi|, \frac{6}{\pi} \frac{\left|\gamma_{k_{0}}\right|}{\sqrt{N}}\right\} .
\end{aligned}
$$

On the other hand, if $|\xi+\pi / 2| \leq 3\left|\gamma_{k_{0}}\right| / \sqrt{N}$, we have

$$
e^{-i \xi}-z_{N, k}=-\left(\frac{\xi}{2}+\frac{\pi}{4}\right)-\frac{\bar{\gamma}_{k}}{\sqrt{N}}+O\left(\frac{1}{N}\right)
$$

hence

$$
\left|e^{-i \xi}-z_{N, k}\right| \geq \frac{1}{2} \frac{\inf \operatorname{Im} \gamma_{k}}{\sqrt{N}}=\frac{c_{0}}{\sqrt{N}} \geq C_{0} \max \left\{\frac{1}{\sqrt{N}}, \frac{1}{6\left|\gamma_{k_{0}}\right|}|\cos \xi|\right\} .
$$


Thus we have obtained

$$
\begin{aligned}
\mid \frac{d}{d \xi} \omega\left(z_{N, 1}, \ldots, z_{N, N}\right)(\xi) & -\sum_{k=1}^{N} \operatorname{Im} \frac{i e^{-i \xi}}{e^{-i \xi}-Z_{N, k}} \mid \\
& \leq C \sum_{k=1}^{N} \frac{(1+\log k)}{\sqrt{N k}} \frac{1}{\frac{k}{N}+\cos ^{2} \xi} \\
& \leq C \sqrt{N} \sum_{1}^{\infty} \frac{1+\log k}{k \sqrt{k}} .
\end{aligned}
$$

Now we look at

$$
S_{N}(\xi)=\operatorname{Im} \sum_{k=1}^{N} \frac{i e^{-i \xi}}{e^{-i \xi}-Z_{N, k}}
$$

as at a Riemann sum: we have

$$
\frac{\pi}{N} S_{N}(\xi) \underset{N \rightarrow \infty}{\longrightarrow} \operatorname{Im} \int_{0}^{\pi} \frac{i e^{-i \xi} d \omega}{e^{-i \xi}-Z(\omega)} .
$$

If $\xi \neq \pm \pi / 2$, we have a proper Riemann integral; if $\xi= \pm \pi / 2$, the integrand is unbounded at $0(\xi=-\pi / 2)$ or $\pi(\xi=\pi / 2)$; but for $\xi=-\pi / 2$ we have $e^{-i \xi}-Z(\omega)=e^{i \pi / 4} \sqrt{2 \omega}+O(\omega)$ near $\omega=0$ and thus

$$
\int_{0}^{\pi} \frac{1}{|i-Z(\omega)|} d \omega<+\infty
$$

It is easy to evaluate the distance between $\pi S_{N} / N$ and the integral. We have

$$
\begin{gathered}
\left|\int_{0}^{7 \pi /(8 N+6)} \frac{d \omega}{e^{-i \xi}-Z(\omega)}\right| \leq C \int_{0}^{7 \pi /(8 N+6)} \frac{d \omega}{\sqrt{\omega}} \leq C^{\prime} \frac{1}{\sqrt{N}} \\
\left|\int_{(8 N-1) \pi /(8 N+6)}^{\pi} \frac{d \omega}{e^{-i \xi}-Z(\omega)}\right| \leq C \int_{(8 N-1) \pi /(8 N+6)}^{\pi} \frac{d \omega}{\sqrt{\pi-\omega}} \\
\leq C^{\prime} \frac{1}{\sqrt{N}}, \\
\frac{1}{N \mid}\left|\frac{1}{e^{-i \xi}-Z\left(\frac{8 N-1}{8 N+6} \pi\right)}\right| \leq C^{\prime} \frac{\sqrt{N}}{N},
\end{gathered}
$$


and finally for $1 \leq k<N$

$$
\begin{aligned}
& \left|\int_{(8 k-1) \pi /(8 N+6)}^{(8 k+7) \pi /(8 N+6)} \frac{1}{e^{-i \xi}-Z(\omega)} d \omega-\frac{8 \pi}{8 N+6} \frac{1}{e^{-i \xi}-Z\left(\frac{8 k-1}{8 N+6}\right) \pi}\right| \\
& \quad \leq C \int_{(8 k-1) /(8 N+6)}^{(8 k+7) \pi /(8 N+6)} \frac{\left|Z(\omega)-Z\left(\frac{8 k-1}{8 N+6}\right) \pi\right|}{\left|e^{-i \xi}-Z(\omega)\right| \mid e^{-i \xi-Z\left(\frac{8 k-1}{8 N+6} \pi\right) \mid} d \omega} \\
& \quad \leq C^{\prime} \int_{(8 k-1) \pi /(8 N+6)}^{(8 k+7) \pi /(8 N+6)} \frac{\frac{1}{\sqrt{N k}}}{\sqrt{\frac{k}{N}} \sqrt{\frac{k}{N}}} d \omega \\
& \leq C^{\prime \prime} \frac{1}{k^{3 / 2} \sqrt{N}}
\end{aligned}
$$

and thus

$$
\left|\frac{\pi}{N} S_{N}(\xi)-\operatorname{Im} \int_{0}^{\pi} i e^{-i \xi} \frac{d \omega}{e^{-i \xi}-Z(\omega)}\right| \leq C \frac{1}{\sqrt{N}} .
$$

Thus, Result 10 is proved since writing $-e^{-2 i \omega}=e^{-i \sigma}$ gives

$$
\begin{aligned}
& \int_{0}^{\pi} i e^{-i \xi} \frac{d \omega}{e^{-i \xi}-\sqrt{2 \sin \omega} e^{i(\pi / 4-\omega / 2)}-e^{i(\pi / 2-\omega)}} \\
& =\frac{1}{2} \int_{-\pi}^{\pi} i e^{-i \xi} \frac{d \sigma}{e^{-i \xi}-\sqrt{e^{-i \sigma}}-\sqrt{1+e^{-i \sigma}}} .
\end{aligned}
$$

We will easily prove Theorem 2 if we know the value of $I(\xi)=$ $\int_{-\pi}^{\pi} i e^{-i \xi} d \sigma /\left(e^{-i \xi}-\xi(\sigma)\right)$ :

Result 11. Let $\xi(\sigma)=\sqrt{e^{-i \xi}}+\sqrt{1+e^{-i \sigma}}$ and $\xi \in[-\pi, \pi]$. Then

$$
\begin{aligned}
\int_{-\pi}^{\pi} i e^{-i \xi} \frac{d \sigma}{e^{-i \xi}-\xi(\sigma)} \\
\quad= \begin{cases}-\pi \tan \left(\frac{\xi}{2}\right)+i \frac{\cos \xi}{\sin \xi} \log \left(\frac{1-\sin \xi}{1+\sin \xi}\right), & \text { if }|\xi| \leq \frac{\pi}{2} \\
-\pi \operatorname{cotan}\left(\frac{\xi}{2}\right)+i \frac{\cos \xi}{\sin \xi} \log \left(\frac{1-\sin \xi}{1+\sin \xi}\right), & \text { if }|\xi| \geq \frac{\pi}{2}\end{cases}
\end{aligned}
$$


We find that $I(\xi)$ is continuous, which is obvious since by (61)

$$
\left|e^{-i \xi}-\xi(\sigma)\right| \geq C \sqrt{\pi^{2}-\sigma^{2}},
$$

so that we may apply Lebesgue's dominated convergence theorem.

Proof. Since $\xi(\sigma)=\bar{\xi}(-\sigma)$, we find that

$$
I(-\xi)=-\overline{\int_{-\pi}^{\pi} \frac{i e^{-i \xi}}{e^{-i \xi}-\bar{\xi}(\sigma)} d \sigma}=-\overline{I(\xi)},
$$

so that it is enough to compute $I(\xi)$ for $\xi \in[0, \pi]$.

Writing $e^{-i \sigma}=u$, we may write

$$
I(\xi)=\int_{-1+i 0}^{-1-i 0} \frac{e^{-i \xi}}{\sqrt{u}+\sqrt{1+u}-e^{-i \xi}} \frac{d u}{u},
$$

where $u$ runs clockwise on the circle $|u|=1$. The function

$$
f(z)=\frac{e^{-i \xi}}{z\left(\sqrt{z}+\sqrt{1+z}-e^{-i \xi}\right)}
$$

is analytical on $\mathbb{C} \backslash(-\infty, 0]$ and may be extended continuously to $(-\infty$, $0]+i 0$ and $(-\infty, 0]-i 0$ but at three points: $z=0$ (both a pole and a branching point), $z=-1$ (a branching point) and if $\xi \in[0, \pi / 2]$ at $-\sin ^{2} \xi-i 0=z_{\xi}$. Thus we may write:

- for $\xi \in[\pi / 2, \pi]$

$$
\begin{aligned}
I(\xi)= & \lim _{\varepsilon \rightarrow 0} \int_{-1}^{-\varepsilon} \frac{e^{-i \xi}}{\sqrt{u+i 0}+\sqrt{1+u}-e^{-i \xi}} \frac{d u}{u} \\
& +\int_{-\varepsilon}^{-1} \frac{e^{-i \xi}}{\sqrt{u-i 0}+\sqrt{1+u}-e^{-i \xi}} \frac{d u}{u} \\
& +\int_{-\varepsilon+i 0}^{-\varepsilon-i 0} \frac{e^{-i \xi}}{\sqrt{u}+\sqrt{1+u}-e^{-i \xi}} \frac{d u}{u} \\
= & 2 i \int_{0}^{1} \frac{d t}{\cos \xi-\sqrt{1-t^{2}}}-2 i \pi \frac{e^{-i \xi}}{1-e^{-i \xi}} \\
= & 2 i \int_{0}^{\pi / 2} \frac{\cos \alpha}{\cos \xi-\cos \alpha} d \alpha-\pi \operatorname{cotan}\left(\frac{\xi}{2}\right)+\pi i .
\end{aligned}
$$


- if $\xi \in(0, \pi / 2)$ we have, writing $t_{\varepsilon}^{+}=\sqrt{\sin ^{2} \xi+\varepsilon}$ and $t_{\varepsilon}^{-}=$ $\sqrt{\sin ^{2} \xi-\varepsilon}$

$$
I(\xi)=\lim _{\varepsilon \rightarrow 0} A_{\varepsilon}+B_{\varepsilon}+C_{\varepsilon},
$$

where

$$
\begin{aligned}
& A_{\varepsilon}= \int_{-1}^{-\left(t_{\varepsilon}^{+}\right)^{2}}+\int_{-\left(t_{\varepsilon}^{-}\right)^{2}}^{-\varepsilon} \frac{e^{-i \xi}}{\sqrt{u+i 0}+\sqrt{1+u}-e^{-i \xi}} \frac{d u}{u} \\
&+\int_{-\varepsilon}^{-\left(t_{\varepsilon}^{-}\right)^{2}}+\int_{-\left(t_{\varepsilon}^{+}\right)^{2}}^{-1} \frac{e^{-i \xi}}{\sqrt{u-i 0}+\sqrt{1+u}-e^{-i \xi}} \frac{d u}{u} \\
&=2 i \int_{\sqrt{\varepsilon}}^{t_{\varepsilon}^{-}}+\int_{t_{\varepsilon}^{+}}^{1} \frac{d t}{\cos \xi-\sqrt{1-t^{2}}} \\
& B_{\varepsilon}=\int_{-\varepsilon+i 0}^{-\varepsilon-i 0} \frac{e^{-i \xi}}{\sqrt{u}+\sqrt{1+u}-e^{-i \xi}} \frac{d u}{u} \\
&=-2 i \pi \frac{e^{-i \xi}}{1-e^{-i \xi}+O(\sqrt{\varepsilon})} \\
&=-\pi \operatorname{cotan}\left(\frac{\xi}{2}\right)+i \pi+O(\sqrt{\varepsilon}) \\
& C_{\varepsilon}=\int_{-\left(t_{\varepsilon}^{+}\right)^{2}}^{-\left(t_{\varepsilon}^{-}\right)^{2}} \frac{e^{-i \xi}}{\sqrt{u+i 0}+\sqrt{1+u}-e^{-i \xi}} \frac{d u}{u} \\
&+\int_{z_{\xi}+\varepsilon}^{z_{\xi}-\varepsilon} \frac{e^{-i \xi} d u}{\left(\sqrt{u}+\sqrt{1+u}-e^{-i \xi}\right) u} \\
&=-i \pi 2 i \operatorname{cotan} \xi+O(\varepsilon) \\
&=2 \pi \operatorname{cotan} \xi+O(\varepsilon)
\end{aligned}
$$

since the residue of

$$
f(\xi)=\frac{e^{-i \xi}}{\sqrt{u}+\sqrt{1+u}-e^{-i \xi}} \frac{1}{u}
$$

at $z_{\xi}=-\sin ^{2} \xi-i 0$ is equal to

$$
\frac{e^{-i \xi}}{\frac{1}{2} \frac{1}{\sqrt{z_{\xi}}}+\frac{1}{2} \frac{1}{\sqrt{1+z_{\xi}}}} \frac{1}{z_{\xi}}=\frac{2 \sqrt{z_{\xi}} \sqrt{1+z_{\xi}}}{z_{\xi}}=2 i \operatorname{cotan} \xi
$$


Hence we have

$$
\begin{aligned}
I(\xi)= & \pi\left(2 \operatorname{cotan} \xi-\operatorname{cotan}\left(\frac{\xi}{2}\right)\right) \\
& +i \pi+2 i \lim _{\varepsilon \rightarrow 0} \int_{0}^{t_{\varepsilon}^{-}}+\int_{t_{\varepsilon}^{+}}^{1} \frac{d t}{\cos \xi-\sqrt{1-t^{2}}} \\
= & -\pi \tan \left(\frac{\xi}{2}\right)+i \pi+2 i \lim _{\varepsilon \rightarrow 0} \int_{0}^{\alpha_{\varepsilon}^{-}}+\int_{\alpha_{\varepsilon}^{+}}^{\pi / 2} \frac{\cos \alpha d \alpha}{\cos \xi-\cos \alpha},
\end{aligned}
$$

where $\alpha_{\varepsilon}^{-}=\arcsin t_{\varepsilon}^{-}$and $\alpha_{\varepsilon}^{+}=\arcsin t_{\varepsilon}^{+}$.

Thus, for proving Result 11, we just have to estimate for $\xi \in(0, \pi)$, $\xi \neq \pi / 2$

$$
A(\xi)=\lim _{\varepsilon \rightarrow 0} \int_{0}^{\alpha_{\varepsilon}^{-}}+\int_{\alpha_{\varepsilon}^{+}}^{\pi / 2} \frac{\cos \alpha d \alpha}{\cos \xi-\cos \alpha}
$$

with $\alpha_{\varepsilon}^{-}=\arcsin \sqrt{\sin ^{2} \xi-\varepsilon}$ and $\alpha_{\varepsilon}^{+}=\arcsin \sqrt{\sin ^{2} \xi+\varepsilon}$. We do the usual change of variable $\beta=\tan (\alpha / 2)$. Then

$$
A(\xi)=\lim _{\varepsilon \rightarrow 0} \int_{0}^{\beta_{\varepsilon}^{-}}+\int_{\beta_{\varepsilon}^{+}}^{1} \frac{2\left(1-\beta^{2}\right)}{\left(1+\beta^{2}\right)\left(\left(1+\beta^{2}\right) \cos \xi-\left(1-\beta^{2}\right)\right)} d \beta .
$$

We write

$$
\begin{aligned}
\left(1+\beta^{2}\right) \cos \xi-\left(1-\beta^{2}\right) & =\beta^{2}(1+\cos \xi)-(1-\cos \xi) \\
& =2 \beta^{2} \cos ^{2}\left(\frac{\xi}{2}\right)-2 \sin ^{2}\left(\frac{\xi}{2}\right)
\end{aligned}
$$

hence

$$
\begin{aligned}
& A(\xi)= \frac{1}{\cos ^{2}\left(\frac{\xi}{2}\right)} \lim _{\varepsilon \rightarrow 0} \int_{0}^{\beta_{\varepsilon}^{-}}+\int_{\beta_{\varepsilon}^{+}}^{1} \frac{1-\beta^{2}}{\left(1+\beta^{2}\right)\left(\beta^{2}-\tan ^{2}\left(\frac{\xi}{2}\right)\right)} d \beta \\
&=\frac{1}{\cos ^{2}\left(\frac{\xi}{2}\right)} \lim _{\varepsilon \rightarrow 0} \int_{0}^{\beta_{\varepsilon}^{-}}+\int_{\beta_{\varepsilon}^{+}}^{1}\left(\frac{-2}{1+\tan ^{2}\left(\frac{\xi}{2}\right)} \frac{1}{1+\beta^{2}}\right. \\
&\left.+\frac{1-\tan ^{2}\left(\frac{\xi}{2}\right)}{1+\tan ^{2}\left(\frac{\xi}{2}\right)} \frac{1}{\beta^{2}-\tan ^{2}\left(\frac{\xi}{2}\right)} d \beta\right)
\end{aligned}
$$




$$
\begin{aligned}
& =\lim _{\varepsilon \rightarrow 0} \int_{0}^{\beta_{\varepsilon}^{-}}+\int_{\beta_{\varepsilon}^{+}}^{1}\left(\frac{-2}{1+\beta^{2}}\right. \\
& \left.+\frac{\cos \xi}{\sin \xi}\left(\frac{1}{\beta-\tan \left(\frac{\xi}{2}\right)}-\frac{1}{\beta+\tan \left(\frac{\xi}{2}\right)}\right)\right) d \beta \\
& =\lim _{\varepsilon \rightarrow 0}-\frac{\pi}{2}+\frac{\cos \xi}{\sin \xi} \log \left|\frac{1-\tan \left(\frac{\xi}{2}\right)}{1+\tan \left(\frac{\xi}{2}\right)}\right| \\
& -\frac{\cos \xi}{\sin \xi} \log \left|\frac{\beta_{\varepsilon}^{+}-\tan \left(\frac{\xi}{2}\right)}{\beta_{\varepsilon}^{+}+\tan \left(\frac{\xi}{2}\right)}\right|+\frac{\cos \xi}{\sin \xi} \log \left|\frac{\beta_{\varepsilon}^{-}-\tan \left(\frac{\xi}{2}\right)}{\beta_{\varepsilon}^{-}+\tan \left(\frac{\xi}{2}\right)}\right| \\
& =-\frac{\pi}{2}+\frac{\cos \xi}{2 \sin \xi} \log \left(\frac{1-\tan \left(\frac{\xi}{2}\right)}{1+\tan \left(\frac{\xi}{2}\right)}\right)^{2} \\
& +\frac{\cos \xi}{\sin \xi} \lim _{\varepsilon \rightarrow 0} \log \left|\frac{\beta_{\varepsilon}^{-}-\tan \left(\frac{\xi}{2}\right)}{\beta_{\varepsilon}^{+}-\tan \left(\frac{\xi}{2}\right)}\right| .
\end{aligned}
$$

Now we have

$$
\begin{aligned}
\left(\frac{1-\tan \left(\frac{\xi}{2}\right)}{1+\tan \left(\frac{\xi}{2}\right)}\right)^{2} & =\frac{\cos ^{2}\left(\frac{\xi}{2}\right)-2 \sin \left(\frac{\xi}{2}\right) \cos \left(\frac{\xi}{2}\right)+\sin ^{2}\left(\frac{\xi}{2}\right)}{\cos ^{2}\left(\frac{\xi}{2}\right)+2 \sin \left(\frac{\xi}{2}\right) \cos \left(\frac{\xi}{2}\right)+\sin ^{2}\left(\frac{\xi}{2}\right)} \\
& =\frac{1-\sin \xi}{1+\sin \xi},
\end{aligned}
$$

while we have for $\xi \in(0, \pi / 2)$

$$
\begin{aligned}
\beta_{\varepsilon}^{-}-\tan \left(\frac{\xi}{2}\right) & \sim \frac{1}{2}\left(1+\tan ^{2}\left(\frac{\xi}{2}\right)\right)\left(\alpha_{\varepsilon}^{-}-\xi\right) \\
& \sim \frac{1}{2}\left(1+\tan ^{2}\left(\frac{\xi}{2}\right)\right) \frac{\sqrt{\sin ^{2} \xi-\varepsilon}-\sin \xi}{\cos \xi} \\
& \sim \frac{-\varepsilon\left(1+\tan ^{2}\left(\frac{\xi}{2}\right)\right)}{4 \sin \xi \cos \xi}
\end{aligned}
$$


and

$$
\beta_{\varepsilon}^{+}-\tan \left(\frac{\xi}{2}\right) \sim \frac{\left.+\varepsilon\left(1+\tan ^{2} \frac{\xi}{2}\right)\right)}{4 \sin \xi \cos \xi} \sim-\left(\beta_{\varepsilon}^{-}-\tan \left(\frac{\xi}{2}\right)\right) .
$$

Thus

$$
A(\xi)=-\frac{\pi}{2}+\frac{\cos \xi}{2 \sin \xi} \log \frac{1-\sin \xi}{1+\sin \xi}
$$

and Result 11 is proved.

Now, (63) gives

$$
\left|\frac{d}{d \xi} \omega\left(z_{N, 1}, \ldots, z_{N, N}\right)(\xi)-\frac{N}{2 \pi} \frac{\cos \xi}{\sin \xi} \log \frac{1-\sin \xi}{1+\sin \xi}\right| \leq C \sqrt{N} .
$$

Integrating this for $\xi \in[-\pi, \pi]$ we get

$$
\left|\omega\left(z_{N, 1}, \ldots, z_{N, N}\right)(\xi)-\frac{N}{2 \pi}\left(\operatorname{Li}_{2}(-\sin \xi)-\operatorname{Li}_{2}(\sin \xi)\right)\right| \leq C \sqrt{N} .
$$

Since both functions are $2 \pi$-periodical, this inequality can be extended to all $\xi \in \mathbb{R}$ and Theorem 2 is proved.

\section{Almost linear-phased Daubechies filters.}

In this section, we prove Theorem 3. The proof is very easy. Indeed, we want to estimate for $N=4 q, \omega\left(z_{N, 1}^{\varepsilon_{N, 1}}, \ldots, z_{N, N}^{\varepsilon_{N, N}}\right)(\xi)$ with $\varepsilon_{N, k}=1$ if $k=0 \bmod 4$ or $k=1 \bmod 4$, and $\varepsilon_{N, k}=-1$ otherwise.

We have (writing $\omega_{N}$ for $\omega\left(z_{N, 1}^{\varepsilon_{N, 1}}, \ldots, z_{N, N}^{\varepsilon_{N, N}}\right), K_{N}$ for $\{k \in \mathbb{N}: 1 \leq$ $\left.k \leq N, \varepsilon_{N, k}=1\right\}$ and $\tilde{K}_{N}$ for $\left.\left\{k \in \mathbb{N}: 1 \leq k \leq N, \varepsilon_{N, k}=-1\right\}\right)$

$$
\frac{d \omega_{N}}{d \xi}=\operatorname{Im} \sum_{k \in K_{N}} \frac{i e^{-i \xi}}{e^{-i \xi}-z_{N, k}}+\sum_{k \in \tilde{K}_{N}} \frac{i e^{-i \xi}}{e^{-i \xi}-\frac{1}{\bar{z}_{N, k}}}
$$

(we have used that for $k \in \tilde{K}_{N}, N+1-k \in \tilde{K}_{N}$ and $z_{N, k}=\bar{z}_{N, N+1-k}$ ). Hence we have

$$
\begin{aligned}
\frac{d \omega_{N}}{d \xi}= & \operatorname{Im}\left(\sum_{k \in K_{N}} \frac{i e^{-i \xi}}{e^{-i \xi}-z_{N, k}}-\sum_{k \in \tilde{K}_{N}} \frac{i e^{-i \xi}}{e^{-i \xi}-z_{N, k}}\right) \\
& +\operatorname{Im}\left(\sum_{k \in \tilde{K}_{N}} \frac{i e^{-i \xi}}{e^{-i \xi}-z_{N, k}}+\frac{i e^{-i \xi}}{e^{-i \xi}-\frac{1}{\bar{z}_{N, k}}}\right)
\end{aligned}
$$


But we have

$$
\begin{aligned}
\frac{i e^{-i \xi}}{e^{-i \xi}-Z}+\frac{i e^{-i \xi}}{e^{-i \xi}-\frac{1}{\bar{Z}}} & =\frac{i e^{-i \xi}}{e^{-i \xi}-Z}+\frac{i \bar{Z}}{\bar{Z}-e^{+i \xi}} \\
& =\frac{i e^{-i \xi}\left(e^{i \xi}-\bar{Z}\right)+i \bar{Z}\left(-e^{-i \xi}+Z\right)}{\left|e^{-i \xi}-Z\right|^{2}} \\
& =\frac{i\left(1-2 \bar{Z} e^{-i \xi}+|Z|^{2}\right)}{\left|Z-e^{-i \xi}\right|^{2}} \\
& =i+\frac{i\left(Z e^{i \xi}-\bar{Z} e^{-i \xi}\right)}{\left|Z-e^{-i \xi}\right|^{2}}
\end{aligned}
$$

hence

$$
\operatorname{Im}\left(\frac{i e^{-i \xi}}{e^{-i \xi}-Z}+\frac{i e^{-i \xi}}{e^{-i \xi}-\frac{1}{\bar{Z}}}\right)=1
$$

Thus, we have obtained

$$
\begin{aligned}
\frac{d \omega_{N}}{d \xi}=\frac{N}{2}+\operatorname{Im} \sum_{k=1}^{q} i e^{-i \xi} & \left(\frac{1}{e^{-i \xi}-z_{N, 4 k-3}}-\frac{1}{e^{-i \xi}-z_{N, 4 k-2}}\right. \\
& \left.-\frac{1}{e^{-i \xi}-z_{N, 4 k-1}}+\frac{1}{e^{-i \xi}-z_{N, 4 k}}\right) .
\end{aligned}
$$

Now we write, for $r \in\{1,2,3\}$

$$
\begin{aligned}
\frac{1}{e^{-i \xi}-z_{N, 4 k-r}}= & \frac{1}{e^{-i \xi}-z_{N, 4 k}}+\frac{z_{N, 4 k-r}-z_{N, 4 k}}{\left(e^{-i \xi}-z_{N, 4 k}\right)\left(e^{-i \xi}-z_{N, 4 k-r}\right)} \\
= & \frac{1}{e^{-i \xi}-z_{N, 4 k}}+\frac{z_{N, 4 k-r}-z_{N, 4 k}}{\left(e^{-i \xi}-z_{N, 4 k}\right)^{2}} \\
& +\frac{\left(z_{N, 4 k-r}-z_{N, 4 k}\right)^{2}}{\left(e^{-i \xi}-z_{N, 4 k}\right)^{2}\left(e^{-i \xi}-z_{N, 4 k-r}\right)} .
\end{aligned}
$$

We have, writing $\tilde{k}=\min \{k, q+1-k\}$

$$
\left|\frac{\left(z_{N, 4 k-r}-z_{N, 4 k}\right)^{2}}{\left(e^{-i \xi}-z_{N, 4 k}\right)^{2}\left(e^{-i \xi}-z_{N, 4 k-r}\right)}\right| \leq C \frac{\frac{1}{N \tilde{k}}}{\left(\frac{\tilde{k}}{N}+\cos ^{2} \xi\right)^{3 / 2}} \leq \frac{C \frac{1}{\tilde{k}} \sqrt{\frac{1}{N \tilde{k}}}}{\frac{\tilde{k}}{N}+\cos ^{2} \xi}
$$


and

$$
\begin{aligned}
\int_{-\pi}^{\pi} \frac{d \xi}{\frac{\tilde{k}}{N}+\cos ^{2} \xi} & \leq 4 \int_{0}^{\arccos \sqrt{\tilde{k} / N}} \frac{d \xi}{\cos ^{2} \xi}+\frac{4 N}{\tilde{k}} \int_{\arccos \sqrt{\tilde{k} / N}}^{\pi / 2} d \xi \\
& =4 \sqrt{\frac{N}{\tilde{k}}} \sin \left(\arccos \sqrt{\frac{\tilde{k}}{N}}\right)+\frac{4 N}{\tilde{k}} \arcsin \sqrt{\frac{\tilde{k}}{N}} \\
& \leq 4 \sqrt{\frac{N}{\tilde{k}}}+2 \pi \sqrt{\frac{N}{\tilde{k}}}
\end{aligned}
$$

so that

$$
\begin{array}{r}
\int_{-\pi}^{\pi}\left|\frac{d \omega_{N}}{d \xi}-\frac{N}{2}-\operatorname{Im} \sum_{k=1}^{q} \frac{z_{N, 4 k-3}-z_{N, 4 k-2}-z_{N, 4 k-1}+z_{N, 4 k}}{\left(e^{-i \xi}-z_{N, 4 k}\right)^{2}}\right| \\
\leq C \sum_{k=1}^{\infty} \frac{1}{k^{2}}=C^{\prime}<+\infty
\end{array}
$$

and

$$
\begin{aligned}
& \int_{-\pi}^{\pi}\left|\frac{d \omega_{N}}{d \xi}-\frac{N}{2}\right| d \xi \\
& \leq C^{\prime}+C \sum_{k=1}^{q} \sqrt{\frac{N}{\tilde{k}}}\left|z_{N, 4 k-3}-z_{N, 4 k-2}-z_{N, 4 k-1}+z_{N, 4 k}\right| .
\end{aligned}
$$

When $\tilde{k} \leq k_{0}$, we write

$$
\left|z_{N, 4 k-r}-z_{N, 4 k+1-r}\right|=O\left(\frac{1}{\sqrt{N \tilde{k}}}\right)
$$

and obtain

$$
\sum_{\tilde{k} \leq k_{0}} \sqrt{\frac{N}{\tilde{k}}}\left|z_{N, 4 k-3}-z_{N, 4 k-2}-z_{N, 4 k-1}+z_{N, 4 k}\right| \leq C \log k_{0} .
$$

When $\tilde{k} \geq k_{0}$, we may write as in formula (58)

$$
\begin{aligned}
z_{N, 4 k-r} & =y_{N, 4 k-r}+\sqrt{y_{N, 4 k-r}^{2}-1}+O\left(\frac{\log \tilde{k}}{\tilde{k} \sqrt{N \tilde{k}}}\right) \\
& =\sqrt{\omega_{N, 4 k-r}}+\sqrt{\omega_{N, 4 k-r}+1}+O\left(\frac{\log \tilde{k}}{\tilde{k} \sqrt{N \tilde{k}}}\right),
\end{aligned}
$$


where

$$
\begin{aligned}
\omega_{N, \ell}= & -e^{-2 i \pi(8 \ell-1) /(8 N+6)} \\
& -\frac{1}{N} e^{-2 i \pi(8 \ell-1) /(8 N+6)} \log \left(2 \sqrt{2 N \pi \sin \left(\frac{8 \ell-1}{8 N+6} \pi\right)}\right) .
\end{aligned}
$$

We write

$$
\sqrt{\alpha+\beta}=\sqrt{\alpha}+\frac{\beta}{\sqrt{\alpha}+\sqrt{\alpha+\beta}}=\sqrt{\alpha}+\frac{\beta}{2 \sqrt{\alpha}}-\frac{\beta^{2}}{2 \sqrt{\alpha}(\sqrt{\alpha}+\sqrt{\alpha+\beta})^{2}}
$$

Now, we have: $\omega_{N, \ell}$ is order of magnitude $1, \omega_{N, \ell}+1$ is of order of magnitude $\min \{\sqrt{\ell / N}, \sqrt{(N+1-\ell) / N}\}$ and $\omega_{N, \ell+1}-\omega_{N, \ell}$ is of order of magnitude $1 / N$. Thus, we may write

$$
\begin{gathered}
\sqrt{\omega_{N, 4 k-r}}=\sqrt{\omega_{N, 4 k}}+O\left(\frac{1}{N}\right) \\
\sqrt{1+\omega_{N, 4 k-r}}=\sqrt{1+\omega_{N, 4 k}}+\frac{\omega_{N, 4 k-r}-\omega_{N, 4 k}}{2 \sqrt{1+\omega_{N, 4 k}}+O\left(\frac{1}{\tilde{k} \sqrt{N \tilde{k}}}\right)} \\
=\sqrt{1+\omega_{N, 4 k}}+\frac{e^{-2 i \pi(32 k-1) /(8 N+6)}\left(1-e^{2 i 8 r \pi /(8 N+6)}\right)}{2 \sqrt{1+\omega_{N, 4 k}}} \\
+O\left(\frac{\log \tilde{k}}{N^{2}}\right)+O\left(\frac{1}{\tilde{k} \sqrt{N \tilde{k}}}\right)
\end{gathered}
$$

and finally

$$
\begin{aligned}
& \frac{\sqrt{N}}{\tilde{k}}\left|z_{N, 4 k-3}-z_{N, 4 k-2}-z_{N, 4 k-1}+z_{N, 4 k}\right| \\
& =\sqrt{\frac{N}{\tilde{k}}}\left|\frac{e^{2 i 24 \pi /(8 N+6)}-e^{2 i 16 \pi /(8 N+6)}-e^{2 i 8 \pi /(8 N+6)}+1}{2 \sqrt{1+\omega_{N, 4 k}}}\right| \\
& \quad+O\left(\frac{\log \tilde{k}}{\tilde{k}^{2}}\right)+O\left(\frac{1}{\sqrt{N \tilde{k}}}\right)+O\left(\frac{\log \tilde{k}}{N \sqrt{N \tilde{k}}}\right) \\
& =O\left(\frac{1}{N \tilde{k}}\right)+O\left(\frac{\log \tilde{k}}{\tilde{k}^{2}}\right)+O\left(\frac{1}{\sqrt{N \tilde{k}}}\right)+O\left(\frac{\log \tilde{k}}{N \sqrt{N \tilde{k}}}\right) .
\end{aligned}
$$


We thus have proved Theorem 3 , since

$$
\begin{gathered}
\sum_{1}^{N} \frac{1}{N \tilde{k}} \leq C \frac{\log N}{N}=o(1), \\
\sum_{1}^{N} \frac{1}{\sqrt{N \tilde{k}}} \leq C \frac{\sqrt{N}}{\sqrt{N}}=C<+\infty, \\
\sum_{1}^{\infty} \frac{\log \tilde{k}}{\tilde{k}^{2}}<+\infty, \\
\sum_{1}^{N} \frac{\log \tilde{k}}{N \sqrt{N \tilde{k}}} \leq C \frac{1}{N \sqrt{N}} \sqrt{N} \log N=o(1) .
\end{gathered}
$$

\section{References.}

[1] Caglar, H., Akansu, A. N., A generalized parametric PR-QMF design technique based on Bernstein polynomial approximation. IEEE Trans. Signal Processing 41 (1993), 2314-2321.

[2] Daubechies, I., Orthonormal bases of compactly supported wavelets. Comm. Pure Appl. Math. 41 (1988), 909-996.

[3] Fettis, H. E., Caslin, J. C., Cramer, K. R., Complex zeros of the error function and of the complementary error function. Math. Comp. 27 (1973), 401-407.

[4] Herrmann, O., On the approximation problem in nonrecursive digital filter design. IEEE Trans. on Circuit Theory (1971), 411-413.

[5] Kantorovich, L. V., Sur la convergence de la suite des polynômes de S. Bernstein en dehors de l'intervalle fondamental. Bull. Acad. Sci. URSS (1931), 1103-1115.

[6] Lemarié-Rieusset, P. G., Polynômes de Bernstein en théorie des ondelettes. C. R. Acad. Sci. Paris 319 (1994), 21-24.

[7] Lorentz, G. G., Bernstein polynomials. Chelsea Publ., 1986.

[8] Meyer, Y., Ondelettes et opérateurs. vol. I. Hermann, 1990.

[9] Nevanlinna, R., Le théorème de Picard-Borel et la théorie des fonctions méromorphes. Gauthier-Villars, 1929.

[10] Pólya, G., Szegö, G., Problems and theorems in Analysis, I. SpringerVerlag, 1986. 
[11] Strichartz, R., How to make wavelets. Amer. Math. Montly 100 (1993), 539-556.

[12] Varga, R. S., Edrei, A., Saff, E. B., Zeros of sections of power series. Lecture Notes in Math. 1002 (1983), Springer-Verlag.

Recibido: 20 de febrero de 1.995

Revisado: 10 de noviembre de 1.995

Djalil Kateb

Université Technologique de Compiègne

Mathématiques

Centre de Royallieu

BP 649

60206 Compiègne Cedex

FRANCE

Djalil.Kateb@dna.utc.fr

and

Pierre Gilles Lemarié-Rieusset

Université d'Evry

Mathématiques

Bd des Coquibus

91025 Evry Cedex

FRANCE

lemarie@lami.univ-evry.fr 